Anuario de Estudios Medievales

42/2, julio-diciembre de 2012, pp. 501-534

ISSN 0066-5061

doi:10.3989/aem.2012.42.2.01

\title{
LA CRISE FINANCIÈRE DES HOSPITALIERS DE RHODES AU QUINZIËME SIÈCLE (1426-1480)
}

\author{
THE FIFTEENTH-CENTURY FINANCIAL CRISIS \\ OF THE HOSPITALLERS OF RHODES (1426-1480)
}

PIERRE BONNEAUD

\begin{abstract}
Résumé: L'Ordre de l'Hôpital installé à l'île de Rhodes depuis 1310 connut une longue et grave crise financière lorsque, à partir de 1426, les attaques des Mamelouks du sultan d'Égypte puis, après 1453, celles des Turcs ottomans à la conquête de la Méditerranée orientale le mirent en grand danger. Il dut dépenser, et pour cela trouver, des sommes considérables pour préparer sa défense. Cet article se propose d'abord de présenter l'organisation des finances de l'Ordre au XVe siècle, autour du Trésor, puis de faire l'inventaire de l'endettement et des mesures prises pour s'efforcer de redresser la situation. Les emprunts avaient pris de multiples formes: censals, lettres de change, prêts usuraires, prêts déguisés, etc. L'Ordre fit largement appel aux marchands d'Occident fréquentant Rhodes, en particulier aux Catalans. Les plans de redressement furent nombreux et entraînèrent l'intervention de la papauté.
\end{abstract}

Mots-clés: Rhodes; chapitres généraux; commanderies; responsiones; papauté; credit; prêts; censals; lettres de change; marchands.

\begin{abstract}
The Order of the Hospital, which was established at Rhodes from 1310 , experienced a long and serious financial crisis as a consequence of several attacks from the Mamluks, from 1426 to 1444 , followed by others from the Ottoman Turks after 1453. The Order had to face heavy expenses to defend itself and needed large supplies of money. This article will describe the Treasury organization of the Order, the extent of its debts and the measures taken to improve the situation. Censals, bills of exchange, usury loans and masked loans were some of the ways used to raise funds. The Order requested financial support from the Western merchants who were established at Rhodes, especially from the Catalans. There were many recovery plans, in some cases with the arbitration of the Papacy.
\end{abstract}

Keywords: Rhodes; general chapters; commanderies; responsiones; papacy; credit; loans; censals; bills of exchange; merchants.

\footnotetext{
${ }^{1}$ Abréviations utilisées: $\mathrm{ACA}=$ Arxiu de la Corona d'Aragó; $\mathrm{ADHG}=$ Archives Départementales de la Haute-Garonne; AGP = Arxiu del Gran Priorat; AHCB = Arxiu Històric de la Ciutat de Barcelona; $\mathrm{AOM}=$ Archives of the Order of Malta; ASV = Archivio Segreto Vaticano; $\mathrm{RC}=$ Registre de Chancellerie; Reg. Vat. = Registra Vaticana.
} 


\section{SOMMAIRE}

1. Introduction.- 2. Les finances de l'Hôpital face à la crise.- 2.1. L'organisation des finances de l'Ordre au milieu du XVe siècle.- 2.2. Les origines de la crise financière des Hospitaliers de Rhodes.- 2.3. Les divisions au couvent face à la crise financière.- 3. Le recours à l'emprunt: la dette sous toutes ses formes.- 3.1. L'ampleur de la dette.- 3.2. Le recours aux censals de Barcelone.- 3.3. Les lettres de change.- 3.4. Les prêts à financier intérêt des marchands à Rhodes.3.5. Prêts dissimulés et autres formes de crédit.- 3.6. Le rôle financier des marchands, contesté mais indispensable.- 4. L'accroissement des ressources.- 4.1. La surtaxation des commanderies.- 4.2. L'administration du couvent et du Trésor confiée au maître.- 4.3. Autres apports.5. Plus de rigueur dans la prévision et dans le contrôle des dépenses.- 5.1. La transparence face à la crise.- 5.2. L'exemple du chapitre général de Rome de 1466.- 5.3. La réduction des dépenses.- 6. Conclusion.- 7. Bibliographie.

\section{INTRODUCTION}

Après la prise d'Acre par les Mamelouks en 1291 et l'expulsion de Palestine des Ordres militaires, suivis d'un bref et insatisfaisant repli à Chypre, l'Ordre de l'Hôpital avait installé son couvent en 1310 à l'île de Rhodes conquise aux dépens de l'empereur de Constantinople avec les principales îles de l'archipel du Dodécanèse ${ }^{2}$. Puis, en 1312, l'Ordre avait obtenu du pape Clément V de recueillir le patrimoine du Temple dont la dissolution venait d'être prononcée. Le maître Foulques de Vilaret avait su convaincre le pontife que, situés à quelques encablures de l'Anatolie, les Hospitaliers pourraient depuis leur place forte maritime continuer la lutte contre les infidèles et assurer une forme de police chrétienne en Méditerranée orientale, en attendant de pouvoir reprendre pied en Terre Sainte ${ }^{3}$.

Comme le Temple était nettement plus riche que lui, l'Hôpital apparut alors comme doté d'un patrimoine considérable, provenant de donations et d'acquisitions dans tout l'Occident, patrimoine géré dans un millier de commanderies regroupées en vingt-trois divisions administratives, les prieurés, avec à la tête de chacun d'eux un prieur $^{4}$. Dans le Levant, l'Ordre avait aussi hérité de biens importants dans le royaume de Chypre.

Les Hospitaliers connurent à Rhodes pendant plus de deux siècles, jusqu'à leur expulsion par les Turcs ottomans en 1523, une expérience bien différente de celle qui avait été la leur en Palestine et en Syrie où ils avaient eu avant tout la terre et le désert comme horizon et comme champs de bataille. À Rhodes et dans les îles qui leur étaient soumises leur univers était devenu absolument et exclusivement maritime. Alors qu'en Palestine ils jouaient leur rôle dans le cadre du royaume de Jerusalem et des principautés latines, ils étaient dorénavant devenus leurs propres maîtres, placés sous la seule autorité du pape et constitués en état religieux souverain avec tous les attributs qui en découlaient. À Rhodes était dorénavant situé le gouvernement de l'Ordre autour du maître et des dignitaires de son conseil. Les prieurés d'Occident,

2 On désignait par « couvent » l'ensemble des frères réunis en Orient autour du Maître de l'Ordre. À Rhodes, le collachium installé dans l'enceinte de l'ancien château byzantin, rassemblait le palais du maître, l'hôpital, l'église Saint-Jean puis les auberges des différentes langues et des maisons diverses.

${ }^{3}$ A. Luttrell, Studies on the Hospitallers, V, pp. 595-622; A. Demurger. Chevaliers du Christ, pp. 226-230 et 234-254.

${ }^{4}$ Ces vingt-trois prieurés incluaient la Castellania d'Amposta qui était en fait le prieuré d'Aragon auquel étaient adjointes les quatre commanderies catalanes de Miravet, Ascó, Horta et Ulldecona situées à l'Ouest du cours de l'Ébre. 
avec les commanderies qui en dépendaient, étaient donc totalement soumis aux décisions prises dans le Levant par ce gouvernement central.

$\mathrm{Au}$ XIVe siècle les activités militaires des Hospitaliers de Rhodes avaient consisté à lancer des attaques ponctuelles sur mer contre les principautés turques d'Anatolie ainsi qu'à être partenaires dans des expéditions contre ces mêmes princes ou contre le sultanat Mamelouk, aux côtés de la papauté, de Venise ou du roi de Chypre $^{5}$. Sur terre, le pape avait confié à l'Hôpital le maintien de la garnison du château du port de Smyrne, conquis en 1344 puis perdu en 1402 lorsque Tamerlan se rendit provisoirement maître de l'Anatolie. L'Hôpital construisit alors, sur le sol même de l'Anatolie sur l'ancien site d'Halicarnasse et en face de l'île de Cos, la forteresse du château Saint-Pierre aux alentours de $1409^{6}$. L'Ordre chercha aussi sans succès durable à prendre position en Grèce continentale, en Achaïe et dans le Péloponnèse.

Â partir du deuxième quart du XVe siècle la situation des Hospitaliers de Rhodes connut un changement radical face aux Musulmans car les Mamelouks d'Égypte puis les Turcs Ottomans exercèrent une pression sans relâche contre l'Hôpital, avec une succession d'incursions et de sièges, jusqu'à son expulsion de Rhodes en 1523. Les étapes de cette longue période de guerre furent d'abord l'invasion de Chypre par les Mamelouks et la capture du roi Janus en 1426. Rhodes apparut alors comme la cible suivante et prépara sa défense. Une attaque se produisit en 1440, puis un siège de 40 jours en 1444. Les Mamelouks furent repoussés mais demeuraient menaçants ${ }^{7}$.

Après la chute de Constantinople en 1453, le danger vint des Turcs Ottomans qui, après avoir conquis une partie des Balkans et du bassin du Danube, entendaient dominer l'ensemble de la Grèce et de la mer Égée. Ayant pris le contrôle des autres émirats de l'Anatolie et se trouvant à quelques kilomètres de Rhodes, ils exigèrent du maître Lastic, qui refusa, un tribut annuel de 2000 ducats. L'Hôpital et le sultan Mehmed II se trouvèrent alors dans une situation de guerre non déclarée, entraînant à Rhodes et dans l'Archipel un état d'alerte permanent et des mesures de défense face aux incursions fréquentes et aux destructions auxquelles se livraient les vaisseaux ottomans ${ }^{8}$. Rhodes participa, à la mesure de ses moyens, à la croisade conduite sans succès durable par le pape Calixte III entre 1455 et 1458, puis, avec seulement deux galères, en 1472 et 1473, à la ligue éphémère rassemblant des vaisseaux du pape Sixte IV, du roi de Naples et de Venise en guerre contre le Sultan. Lorsque Venise, après avoir perdu la guerre, dut pactiser avec le Sultan celui-ci considéra que le moment était venu d'en terminer avec Rhodes. En 1480, une flotte ottomane de 150 bâtiments entreprit le siège de la ville et débarqua dans l'île. Mais, après trois mois de durs combats les Turcs durent se résoudre à l'échec et lever le camp. Ce succès de l'Hôpital, fortement «médiatisé » par le maître Pierre d'Aubusson fut suivi de plusieurs années de paix, grâce en partie à une crise dynastique à Constantinople à la mort de Mehmed II. Les affrontements reprirent sur mer au XVIe siècle et conduisirent au deuxième siège de Rhodes par les Ottomans entraînant le départ des Hospitaliers en 1523, après plus de deux siècles de présence 9 .

C'est dans le cadre de cette situation de belligérance et d'alerte que se produisit la grave crise financière qui affecta l'ordre de l'Hôpital à Rhodes et dont nous nous proposons d'analyser le déroulement entre les deux dates clefs de 1426 et 1480 , qui marquent le début et l'arrêt, temporaire, de la menace musulmane contre les Hos-

\footnotetext{
${ }^{5}$ A. Luttrell, The Hospitallers of Rhodes, II, pp. 80-116.

${ }^{6}$ A. Luttrell, The Hospitaller State on Rhodes, VI.

${ }^{7}$ L. Nicolau d'Olwer, Un témoignage catalan.

${ }^{8}$ R. Valentini, L'Egeo dopo la caduta di Costantinopoli.

${ }^{9}$ N. Vatin, L'Ordre de Saint-Jean de Jérusalem.
} 
pitaliers de Rhodes. Mais il convient tout d'abord d'examiner les systèmes de gestion des finances de l'Ordre.

\section{LES FINANCES DE L'HÔPITAL FACE À LA CRISE}

Comme toutes les activités du couvent, la gestion des finances s'inscrivait dans un système collégial et oligarchique de gouvernement sous la conduite du maître qui, élu à vie, ne pouvait guère prendre de décision sans son Conseil et sans se soumettre à la tenue des chapitres généraux réunis en principe tous les cinq ans. Ces chapitres prenaient ou ratifiaient les décisions essentielles et, par l'adoption de statuts, ils constituaient un véritable corps législatif ainsi que le pouvoir interne suprême de l'Ordre ${ }^{10}$.

Il faut aussi mettre l'accent sur le caractère multinational de la communauté hospitalière de Rhodes dû à la multiplicité des provenances des commandeurs et frères qui la composaient. Les membres des différents prieurés d'Occident présents à Rhodes y étaient regroupés en entités selon les origines et les prieurés, les Langues, sortes de corporations au nombre de sept au début du siècle, puis huit à partir de $1462^{11}$. Chaque Langue était représentée au Conseil du maître par un bailli conventuel et disposait de son auberge, centre de réunion et de vie collective ${ }^{12}$. Les réunions y étaient nombreuses et les débats, voire les querelles, étaient fréquentes entre les Langues. Au cours du XIVe siècle les trois Langues dites françaises, celles de France, de Provence et d'Auvergne, avaient dominé la conduite des affaires et bénéficié de privilèges dans l'accès aux meilleures dignités, situation que n'acceptaient plus les Langues dites mineures, celles d'Espagne, d'Italie, d'Angleterre et d'Allemagne. Il en résultait des conflits particulièrement aigus notamment dans la gestion des finances de l'Ordre.

\subsection{L'organisation des finances de l'Ordre au milieu du XVe siècle}

L'information sur les finances souffre de la perte de sources spécifiques consacrées à ce sujet, les archives de ce que l'on nommait le Trésor de l'Ordre. Notre connaissance en la matière, très composite, résulte surtout des fonds d'Archives de l'Ordre conservés aux Archives de l'Ordre de Malte à La Valette dont l'essentiel est constitué pour le XVe siècle par les registres de Chancellerie, les comptes rendus des chapitres généraux puis, à partir de 1459, ceux des réunions du Conseil, le recueil des bulles pontificales concernant l'Ordre et des parchemins et manuscrits divers ${ }^{13}$.

${ }^{10}$ J. Sarnowsky, The Oligarchy at Work.

${ }^{11}$ J. Sarnowsky, Der Konvent auf Rhodos. Les sept Langues étaient celles de France, Provence, Auvergne, Espagne, Italie, Angleterre et Allemagne. A partir de 1462, la Langue d'Espagne fut scindée en deux, la Langue d'Aragon, Catalogne et Navarre, d'une part, et la Langue de Castille et Portugal, de l'autre.

${ }_{12}$ Les baillis conventuels étaient: le grand commandeur pour la Langue de Provence, le maréchal, pour celle d'Auvergne, l'infirmier pour celle de France, le drapier pour celle d'Espagne, puis d'Aragon, l'amiral pour celle d'Italie, le turcopolier pour celle d'Angleterre, le grand bailli pour celle d'Allemagne, puis, lorsque la Langue d'Espagne fut scindée en deux, le chancelier pour la Langue de Castille-Portugal.

${ }^{13}$ L'histoire de l'ordre de l'Hôpital écrite au XVIIe siècle par J. Bosio, Dell'Istoria, est fiable en ce qui concerne la période 1437-1480 et comporte de nombreuses informations sur la crise financière de l'Ordre à cette époque. Les principaux ouvrages et articles récents traitant de l'organisation financière des Hospitaliers de Rhodes au XVe siècle sont dus à J. Sarnowsky, Macht und Herrschaft, pp-468-582; idem, The Rights of the Treasury, pp. 267-274. 


\subsubsection{Le rôle des commanderies}

L'ordre de l'Hôpital adoptait des règles nombreuses et complexes de gestion de ses finances, généralement lors des chapitres généraux grâce auxquels nous connaissons son organisation financière centrale. On ne trouve toutefois que peu d'informations sur les finances des commanderies ${ }^{14}$. En effet une grande latitude était laissée dans ce domaine aux commandeurs à condition qu'ils respectent leurs obligations au premier rang desquelles la conservation du patrimoine, l'entretien des églises, la nourriture et l'habillement des frères et des prêtres faisant partie de la commanderie et, d'une manière générale, la prise en charge des dépenses afférentes au bon fonctionnement de ces commanderies.

Mais aux yeux du couvent de Rhodes l'essentiel était leur participation financière à la mission de l'Ordre en Palestine puis dans le Levant en versant régulièrement les contributions annuelles qui leur étaient imposés, appelées responsiones, dont le montant en période normale pouvait être estimé aux alentours de $25 \%$ des revenus de la commanderie. Pendant longtemps les responsiones furent remises aux prieurs lors des chapitres provinciaux annuels mais, à partir du milieu du XIVe siècle, le couvent institua dans chaque prieuré un receveur du Trésor chargé de percevoir ces contributions et de les faire parvenir à Rhodes. Nommés directement par le maître de qui ils recevaient leurs instructions, les receveurs assistaient aux chapitres généraux de l'Ordre mais ils devaient aussi rendre compte de leur gestion au chapitre provincial de leur prieuré. Les commandeurs étaient fréquemment de mauvais payeurs de leurs responsiones et l'intervention constante des receveurs était indispensable pour faire rentrer les arriérés, conséquence des défaillances de commandeurs à régler ponctuellement leurs contributions à la Saint-Jean de chaque année. Lors du chapitre général de Rhodes de 1449 le montant des responsiones de l'ensemble des prieurés à recevoir par le Trésor fut évalué à 50000 florins de Rhodes pour l'ensemble des commanderies soit $25 \%$ du total des rentes elles-mêmes estimées à 200000 florins $^{15}$.

En dehors des responsiones annuelles, en période de crise ou de guerre, des contributions additionnelles, généralement qualifiés d'annates, étaient levées avec l'accord ou la ratification du chapitre général. Ainsi à la fin du XIVe siècle, le maître Juan Fernández de Heredia avait successivement exigé des prieurés des versements exceptionnels de 10000 florins en 1390, puis, en 1394, 20500 florins payables en quatre ans et enfin, à la veille de sa mort en 1396, 32900 florins $^{16}$.

La mort d'un commandeur avait plusieurs conséquences pour les finances de l'Ordre ${ }^{17}$. De leur vivant, les commandeurs, usufruitiers de la commanderie, étaient autorisés à conserver des biens propres et en particulier le surplus que leur laissaient les rentes de la commanderie, une fois assumés ses frais de fonctionnement et le paiement de ses responsiones. À la mort d'un Hospitalier les biens qu'il laissait, que l'on appelait sa dépouille ou spolia, étaient acquis par le Trésor. Il pouvait s'agir de sommes d'argent importantes ou d'objets de grande valeur notamment dans le cas des maîtres, des hauts dignitaires ou de commandeurs particulièrement bien dotés. Il pouvait aussi s'agir du troupeau du commandeur, d'esclaves, de maisons ou de jardins ou encore d'embarcations. Mais le recueil de ces biens n'allait pas sans difficulté car la dépouille devait d'abord servir à payer les dettes éventuelles du défunt parfois su-

14 P. Bonneaud, Le prieuré de Catalogne, pp. 74-99; M. Bonet, La Orden del Hospital, pp. 156-184; idem, Estructura gubernativa, pp. 43-73.

15 J. Sarnowsky, The Rights of the Treasury, p. 271.

${ }^{16}$ P. Bonneaud, Le prieuré de Catalogne, p. 84.

${ }^{17}$ Ibidem, pp. 86-92.

ANUARIO de Estudios MEdievales, 42/2, julio-diciembre 2012, pp. 501-534 ISSN 0066-5061, doi:10.3989/aem.2012.42.2.01 
périeures à ce qu'il laissait, ce qui fut par exemple le cas du maître Battista Orsini à sa mort en $1476^{18}$. Parfois les biens avaient été détournés ou confisqués par des tiers, éventuellement par d'autres Hospitaliers proches du défunt. Le chapitre général de 1471 chargea deux dignitaires d'enquêter sur l'absence de dépouille du commandeur de La Rochelle, Bertrand Jammeron, alors que l'on savait très bien qu'il avait été riche et mené grand train ${ }^{19}$.

Le couvent percevait également les mortuaria, correspondant à la portion des rentes produites dans une commanderie entre la date du décès du commandeur et la Saint-Jean du 24 juin suivant la mort, qui marquait le terme de l'année fiscale. Enfin, même si le remplaçant du commandeur défunt était immédiatement désigné, la commanderie était mise en fermage pendant deux ans et ses revenus étaient encaissés à ce titre par le Trésor au titre de ce que l'on appelait les vacantes. Dans tous ces cas, le receveur du Trésor dans le prieuré se chargeait des encaissements.

\subsubsection{L'organisation des finances au couvent}

En termes de finances on peut dire que le couvent ne s'intéressait à ses commanderies qu'en fonction de leur capacité et de leur efficacité à lui procurer des rentrées de fonds. Étudier les finances de l'Ordre nous conduit donc à nous concentrer sur leur fonctionnement et leurs organes au niveau central, c'est-à-dire à Rhodes où le Trésor, sous la conduite et le contrôle du maître et de son Conseil, gérait tant les entrées provenant des prieurés d'Occident et des possessions du Levant que les dépenses entraînées par le fonctionnement de la communauté.

Le Trésor connut au cours des XIVe et XVe siècles plusieurs évolutions. Jusqu'au milieu du XIVe le dignitaire qui le dirigeait était le Trésorier, personnage important qui figurait au conseil ordinaire du maître. Toutefois entre 1330 et 1350 son rôle diminua d'importance car il fut soumis au grand commandeur, bailli conventuel de la Langue de Provence, et sans disparaître il perdit son rang de bailli conventuel. Il était traditionnellement choisi parmi les frères de la Langue de France ${ }^{20}$. La gestion des finances apparaissait alors comme un fief des Français, situation qui fut la source de récriminations constantes de la part des Langues dites mineures et provoqua des crises répétitives au cours de la période qui nous intéresse. Le Grand Commandeur et le Trésorier étaient assistés de deux autres détenteurs d'offices, le Conservateur général et le scribe du Trésor, chargés de la tenue et du contrôle des comptes.

A partir de 1440 et au cours de la période difficile que traversa l'Hôpital, attaqué par les Mamelouks d'Égypte puis par les Ottomans, les maîtres Lastic puis Milly qui appartenaient tous les deux à une des trois Langues de la France, celle d'Auvergne, durent céder du terrain au profit des Langues mineures, devenues très revendicatives lors des chapitres généraux ${ }^{21}$. Lors de celui de 1440 il fut décidé que le grand commandeur serait assisté de deux procureurs appartenant à d'autres Langues, nommés pour une période d'un an. En 1446, le chapitre convoqué à Rome par le pape Eugène IV décida que les conservateurs généraux devraient être élus par les chapitres généraux et être issus des différentes Langues. Enfin, il fut décidé par le chapitre géné-

\footnotetext{
${ }_{18}$ J. Bosio, Dell'Istoria, p. 361.

19 AOM 380, ff. 152r-153r.

${ }^{20}$ J. Sarnowsky, The Rights of the Treasury, p. 269.

${ }^{21}$ Voir en particulier à titre d'exemple l'article de R. Valentini, Un capitolo generale. Les querelles entre les langues furent aussi particulièrement violentes au cours du chapitre général tenu à Rhodes en 1459 par le maître Jacques de Milly au point d'en paralyser les débats (AOM 282,
} ff.55r-62r). 
ral de 1449 d'instituer un collège de sept auditeurs représentant chacune des Langues, collège qui contrôlerait une fois par semaine les livres du Trésor ${ }^{22}$. Tel était donc le cadre, désormais partagé entre les Langues, dans lequel étaient gérées les finances de l'Ordre au milieu du XVe siècle ${ }^{23}$.

Comme nous l'avons indiqué, le Trésor disposait en Occident d'un receveur par prieuré qui intervenait notamment dans la collecte et l'envoi à Rhodes des responsiones et annates, dans la mise en fermage des commanderies vacantes et répondait à toutes sortes de réquisitions du maître et du Trésor. Les receveurs des prieurés avaient au XVe siècle l'obligation de se rendre aux chapitres généraux et d'y rendre des comptes. Leur mandat était alors systématiquement remis à disposition du maître qui les confirmait ou les remplaçait. Il faut aussi mentionner le receveur général d'Avignon, véritable plaque tournante de la Trésorerie de l'Hôpital en Occident qui organisait les transferts d'argent vers le Levant et gérait une large part des sommes dues à des marchands et des changeurs devenus créditeurs de l'Ordre.

Ce tableau serait incomplet si l'on passait sous silence le fait que des sommes importantes échappaient au Trésor. Il s'agissait des revenus du maître, seigneur de Rhodes, qui percevait les rentes du patrimoine immobilier et mobilier de l'île ainsi que le produit des taxes et charges imposés aux sujets. Le maître était aussi directement titulaire de chambres magistrales, c'est-à-dire de commanderies, généralement une par prieuré d'Occident ainsi qu'à Chypre. Il confiait ces chambres à des commandeurs qui en percevaient les rentes, lui versaient en contrepartie une pension annuelle et ne rendaient compte qu'à lui de leur gestion. Enfin, il devint habituel de confier au maître pour des périodes plus ou moins longues les îles de l'archipel, notamment Cos et Nissyros, avec le droit d'y nommer des commandeurs et des capitaines et d'y percevoir des responsiones, des pensions ou des fermages. Tous ces revenus du maître étaient gérés hors de tout contrôle du Trésor par le sénéchal, l'officier le plus important de son Hôtel.

\subsection{Les origines de la crise financière des Hospitaliers de Rhodes}

Au cours du XIVe siècle, l'Ordre avait déjà dû faire face à des situations de crise financière. Par exemple en 1320, après les frais engagés pour la conquête de Rhodes et des malversations du maître Foulques de Vilaret, les dettes de l'Hôpital s'élevaient à près de 600000 florins dus essentiellement aux trois banques florentines des Bardi, des Peruzzi et des Acciaiuoli. Lorsque ces mêmes banques firent faillite en 1343 et 1346, l'ordre perdit 360000 florins dont il réclamait encore une partie en $1382^{24}$. Les dernières décennies du XIVe siècle avaient aussi été affectées par de grandes difficultés financières en raison du coût des expéditions en Grèce continentale, notamment lors du paiement de la rançon du maître Juan Fernandez de Heredia capturé en Épire par le despote d'Arta ${ }^{25}$.

Mais par son importance, par sa durée et par ses causes la crise du XVe siècle fut d'une toute autre ampleur. L'état de guerre de fait avec les Mamelouks puis avec les Ottomans provoqua par ses conséquences un déséquilibre profond dans les dépenses et les finances de l'Hôpital. Il est aisé d'en énumérer rapidement les raisons.

\footnotetext{
${ }^{22}$ AOM 501, ff. 285r-286r.

${ }^{23}$ J. Sarnowsky, The Rights of the Treasury, pp. 269-270.

${ }^{24}$ A. Luttrell, Studies on the Hospitallers, VI, pp. 17-24.

${ }^{25}$ A. Luttrell, The Hospitallers in Cyprus, Rhodes, I, pp. 301, 306.
} 
Alors que la guerre se déroulait avant tout en mer, les deux galères armées dont disposait l'Ordre en temps de paix était insuffisantes. Il fallut donc en armer quelques autres et lorsque Rhodes était assiégée faire appel à des galères de corsaires ou à de marchands fréquentant le port de Rhodes ${ }^{26}$. En 1444 lors du siège par les Mamelouks les coûts de huit galères ainsi engagées, avec les équipages et les hommes d'armes, s'élevaient à 17000 écus par mois ${ }^{27}$. L'engagement de mercenaires tant sur mer que pour la défense des îles était une autre lourde source de dépenses. En 1444, le maître Lastic avait dû dépenser la somme considérable de 68000 ducats de Rhodes pour payer pendant quatre mois des mercenaires contre les Mamelouks ${ }^{28}$.

Un autre chapitre était celui de la coûteuse construction de murailles et de fortifications autour des places fortes. Rhodes se transforma au XVe siècle sous l'impulsion du maître Fluvià et de ses successeurs en un modèle de l'architecture militaire avec une qualité et une solidité qui expliquent largement l'échec des sièges des Mamelouks et des Ottomans ${ }^{29}$. Il fallut aussi multiplier les achats d'armement et en particulier d'artillerie pour lesquels, dès 1426, des missions ne cessèrent d'être confiées à des dignitaires de l'Ordre chargés de s'en procurer en Occident.

La recherche et le stockage de vivres et de blé en particulier supposa de nouvelles approches après la chute de Constantinople car la fermeture du Bosphore empêchait désormais Rhodes de se fournir dans les régions septentrionales de la Mer Noire comme cela avait été le cas auparavant. La nécessité de stocker des vivres dans l'éventualité des sièges et de se procurer le grain en Italie du Sud et en Sicile notamment ne pouvait qu'augmenter le poids des dépenses d'approvisionnement ${ }^{30}$. Des ambassades et des missions de hauts dignitaires souvent décidées lors des chapitres généraux, se multiplièrent pour se procurer des munitions et des vivres mais aussi pour réclamer l'aide du pape et des princes, également requis de faire pression sur les commandeurs et les frères pour qu'il accourent au couvent et s'acquittent de leurs responsiones ${ }^{31}$. Ces ambassades, indispensables, coûtaient cher au Trésor de Rhodes.

Mais le chapitre le plus important dans les frais nécessaires à la défense était le rassemblement à Rhodes d'un nombre plus important d'Hospitaliers convoqués d'Occident. Les effectifs, qui se situaient entre 200 et 300 aux alentours de 1420 s'accrurent progressivement jusqu'à 450 en 1480 et 550 au début du XVIe siècle. Ces commandeurs et ces frères, avec leurs serviteurs et leurs chevaux, devaient être nourris et la plupart recevaient des salaires ou des pensions que l'ordre s'efforça de réduire.

Le maître et le couvent durent enfin faire preuve de solidarité envers le roi de Chypre également soumis aux attaques du Sultan. Fluvià contribua en 1426 à hauteur de 15000 florins sous forme de prêt à la rançon du roi Janus puis, en 1437, il prêta 69095 florins supplémentaires au souverain qui ne parvenait pas à payer les tributs que lui avait imposé le Sultan d'Égypte ${ }^{32}$.

Il faut de toute évidence voir dans le problème de financement de ces besoins considérables l'origine de la crise financière de l'ordre de l'Hôpital qui s'éternisa pendant près d'un siècle. Les solutions à trouver pour y porter remède occupèrent

${ }^{26}$ AOM 282, f. $15 \mathrm{v}$.

${ }^{27}$ J. Bosio, Dell'Istoria, vol. II, pp. 220; J. Sarnowsky, Macht und Herschaft, p. 566.

${ }^{28}$ J. Sarnowsky, Macht und Herrschaft, p. 567.

${ }^{29}$ A. Gabriel, La cité de Rhodes.

${ }^{30}$ Voir, par exemple, AOM 367, ff. 212r, 214v (1458); AOM 374, ff. 222r-v, 195v, 226r (14631464), AOM 379, ff. 222r-223v (1470).

${ }^{31}$ Par exemple, AOM 356, ff. 240r-244r, AOM 362, f. 63v, AOM 366, f. 67v, AOM 282, f. 119r

32 J. Bosio, Dell'Istoria, pp. 200, 201 et 208. 
alors tous les esprits et divisèrent profondément les dignitaires du couvent. Les oppositions entre les Langues se multiplièrent ainsi que les critiques envers les maîtres.

\subsection{Les divisions au couvent face à la crise financière}

$\mathrm{Au}$ cours de cette période troublée six maîtres se succédèrent au couvent. Ce furent successivement Antoni de Fluvià (1421-1437), Jean de Lastic (1437-1454), Jacques de Milly (1454-1461), Pere Ramon Sacosta (1461-1467), Battista Orsini (1467-1476) et Pierre d'Aubusson (1476-1503). Fluvià et Sacosta furent tous les deux des Catalans appartenant à la Castellania d'Amposta. Orsini, italien, avait été prieur de Rome alors que Lastic, Milly et Aubusson avaient en commun d'avoir été prieurs d'Auvergne. Alors que la présence de ces trois anciens prieurs d'Auvergne répondait à la traditionnelle mainmise des Français, l'accès de deux Catalans et d'un Italien à l'échelon suprême marquait au contraire l'émergence des Langues mineures.

L'ampleur de la crise et la difficulté des prises de décisions amenèrent le couvent à remettre l'administration du Trésor au maître pour des périodes limitées soit pour trois ans à Fluvià en 1429, à Lastic en 1451, pour la même durée à Milly en 1454 et 1459 et en 1466 pour cinq ans à Sacosta, mandat exercé en fait par Orsini jusqu'en 1471 du fait de la mort en février 1467 de son prédécesseur ${ }^{33}$ Pierre d'Aubusson accepta enfin à son tour, lors du chapitre général de 1478, de se voir remettre l'administration du Trésor. Nous nous réservons d'aborder plus en détail par la suite les implications complexes de la remise de la gestion du Trésor « aux mains du maître ».

Fluvià laissa le souvenir d'un gestionnaire prudent et ses spolia, recueillies par le Trésor, s'élevaient, disait-on, à 200000 écus ${ }^{34}$. Ses successeurs Lastic et Milly pour faire face aux attaques des Mamelouks puis des Ottomans durent prendre des mesures contestées par leurs pairs. L'opposition à ces deux maîtres français se manifesta violemment de la part des Langues mineures notamment lors des six chapitres ou assemblées tenues sous leur magistère avec, pour résultat, l'obtention d'une participation accrue de toutes les Langues à la gestion du Trésor. En 1445, l'hostilité de nombreux dignitaires à l'égard de Lastic et des prélèvements accrus qu'il imposa aux commanderies fut telle que le pape Eugène IV, alerté de ces reproches et de ces divergences, décida de réunir le chapitre général à Rome sous son contrôle et hors de la présence du maître ${ }^{35}$. Il épousa le point de vue des opposants à Lastic au point de faire sienne, dans une missive impitoyable, l'accusation que le maître avait dilapidé le «trésor » laissé par Fluvià ${ }^{36}$.

Les différends entre les Langues s'atténuèrent considérablement lorsque le successeur de Milly, le Catalan Pere Ramon Sacosta, fit adopter par le chapitre général de 1462 la division de la Langue d'Espagne entre celle d'Aragon-Catalogne-Navarre et celle de Castille-Portugal, donnant ainsi un poids plus important aux Langues mineures. Au terme d'une longue période de conflits, l'harmonie entre les dignitaires des différentes Langues devint plus habituelle mais l'hostilité ne céda point nécessairement à l'égard du maître. Sacosta, critiqué pour trop défendre ses propres intérêts et pour imposer face à la crise un remède de cheval, le paiement au Trésor de la totalité de leurs rentes par les commandeurs pendant trois ans. Il reçut du pape Paul II le même traitement que son prédécesseur Lastic. Le chapitre général de 1466 fut convoqué à Rome et dirigé en fait par un archevêque et quatre évêques de la Curie.

${ }^{33}$ J. Sarnowsky, The Rights of the Treasury, pp. 272-274.

${ }^{34}$ Voir P. Bonneaud, Le prieuré de Catalogne, pp. 149 et 151.

${ }_{35}$ R. Valentini, Un capitolo generale, pp. 140-142.

${ }^{36}$ ASV, Reg. Vat. 377, ff. 213r-215v. 
Ce chapitre nous a laissé une précieuse analyse de l'état des finances de l'Ordre et un plan de redressement traitant à la fois de l'apurement de la dette, instaurant un cadre de gestion des dépenses du couvent et donnant des directives pour la réduction des dépenses $^{37}$. Ce plan exceptionnel s'imposa ensuite comme modèle lors des chapitres généraux suivants en 1471,1475 et 1478 . Toutes les décisions prises au cours de ces chapitres, qu'il s'agisse de la gestion des finances, de l'administration du couvent ou des statuts traditionnellement adoptés lors de ces réunions, furent soumises à l'approbation pontificale et firent l'objet de bulles du successeur de Paul II, Sixte IV.

Le magistère de Battista Orsini (1467-1476) et surtout celui de Pierre d'Aubusson (1476-1503), auréolé de la résistance de l'Ordre face aux Ottomans lors du siège de 1480, furent moins conflictuels et l'impact de la crise financière, sans disparaître, s'atténua. Mais la papauté continua à faire entendre sa voix dans la gestion des finances de l'Hôpital. Trois chemins s'imposaient à l'évidence pour faire face à la crise: dans l'immédiat, devant l'insuffisance de trésorerie, le recours à l'emprunt, puis l'augmentation des revenus, principalement en réclamant une participation accrue aux Prieurés occidentaux et à leurs commandeurs, et enfin une administration plus rigoureuse du couvent avec, notamment, un plan drastique de réduction des dépenses.

\section{LE RECOURS À L'EMPRUNT: LA DETTE SOUS TOUTES SES FORMES}

\subsection{L'ampleur de la dette}

L'estimation du volume de la dette pose évidemment la question des monnaies dans lesquelles il convient de l'évaluer. Â Rhodes, la monnaie courante de base était l'aspre mais dans les transactions et les rémunérations il était habituel de se référer au florin de Rhodes équivalent à 20 aspres ou au ducat de Rhodes qui valait 32 aspres. La relation en système décimal du ducat au florin était donc de 1 ducat $=1,61$ florin ou, en équivalence monétaire de l'époque, 1 ducat $=1$ florin et 12 aspres $^{38}$. Par rapport au ducat vénitien la valeur de celui de Rhodes était d'un tiers inférieur.

À partir des années 1460, au cours notamment des chapitres généraux et dans l'examen des problèmes financiers, il devint habituel de s'exprimer en écus du royaume de France, sans doute par souci d'homogénéité et de clarté mais plus peutêtre du fait de l'importance prise par le receveur général d'Avignon dans toutes les transactions financières de l'Ordre. L'écu de France était alors évalué à 2 florins de Rhodes, soit un ducat et 8 aspres ${ }^{39}$.

Après l'attaque des Mamelouks en 1426 contre Chypre, Fluvià décida de s'endetter à hauteur de 100000 florins de Rhodes pour préparer la défense des îles. Il prêta par ailleurs, sur ses deniers personnels, près de 85000 florins au roi de Chypre. Lorsque la guerre devint réelle, avec les attaques de 1440 et le siège de 1444, la belligérance en mer, la poursuite des achats d'armes et les nouvelles constructions de fortifications obligèrent Lastic à s'endetter encore plus lourdement notamment par le recours à des censals à Barcelone. À la veille du chapitre général de Rome de 1446 l'endettement était de 100000 ducats de Rhodes, soit environ 160000 florins $^{40}$. Après 1453 et les raids effectués par les Ottomans sur les îles de l'archipel le maître Jacques de Milly multiplia l'appel aux emprunts et lorsque son successeur, le Catalan Pere

${ }^{37}$ AOM 283, ff. 29v-36r.

${ }^{38}$ Z. Tsirpanlis, Anecdota, p. 147.

${ }^{39}$ AOM 282, f. $117 \mathrm{r}$.

${ }^{40}$ R. Valentini, Un capitolo generale, p. 141. 
Ramon Sacosta, se trouvait encore à Barcelone son lieutenant, Adhémar du Puy, lui enjoignit depuis Rhodes de trouver de toute urgence 150000 florins car le Trésor ne pouvait plus faire face au paiement des lettres de change qui étaient échues ${ }^{41}$.

Lors du chapitre général de Rhodes de 1462 les sommes dues aux différents créanciers s'élevaient à 225342 écus (soit 450684 florins de Rhodes) dont 77122 écus à Rhodes même, 75240 à Avignon où résidait le receveur général du Trésor en Occident, 60000 à Barcelone, 7000 en Angleterre et 6000 à Venise. Compte tenu des fonds nécessaires pour assurer, outre le paiement du capital, celui des interêts et frais financiers (interesse et dampna censualium Barcelona et cambiorum Rhodi) et la subsistance du couvent pendant les trois années à venir, le Trésor devait trouver d'ici-là 306369 écus. $^{42}$

Mais le pic de l'endettement fut atteint en 1466 lors de l'audit réalisé à l'occasion du second chapitre général de Rome en 1466 par les évêques de la Curie chargés par le pape Paul II de mettre de l'ordre dans les finances de l'Hôpital. La dette envers les créanciers atteignait alors 287929 écus, soit 575858 florins de Rhodes, c'est-à-dire plus de dix fois le montant fixé pour les dépenses courantes annuelles du couvent $^{43}$. La localisation de la dette était pour 191120 écus à Avignon, 12250 en Angleterre, 4500 en Castille, 42957 à Rhodes et 40000 en Catalogne sous la forme de censals $^{44}$. Les mesures prises ensuite amorcèrent une diminution puisque l'endettement n'était plus que de 120000 écus en 1471 et 76000 en 1476 pour regrimper à 133000 en 1478 puis retomber à 50749 en $1489^{45}$.

Le recours à l'emprunt se heurtait pour un ordre religieux à l'interdiction par l'Église de toutes opérations de crédit avec recherche d'un profit financier, qualifiées d'usuraires. L'Hôpital chercha donc à se procurer des fonds en recourant à des formules acceptées par l'Église qui voyaient en celles-ci des opérations commerciales, telles les censals, les lettres de change ou des achats et ventes de marchandises avec paiements ou livraisons différés. Mais ces approches techniques ne suffisaient pas et le recours pur et simple aux prêts à intérêt, ad dampna et interesse, c'est-à-dire à caractère d'usure, se multiplièrent. Les marchands occidentaux établis à Rhodes, en particulier les Catalans, jouèrent un rôle primordial dans le financement des besoins de l'Ordre ${ }^{46}$.

Pour étudier ces différentes formules dans la période qui nous intéresse nous disposons d'une part d'un recensement des créances envers l'Hôpital fait par Jürgen Sarnowsky et, d'autre part, de nos propres recherches ${ }^{47}$.

\subsection{Le recours aux censals de Barcelone}

\subsubsection{Les déboires d'Antoni de Fluvià. Un emprunt de l'Ordre détourné au profit du roi d'Aragon}

Peu de temps après l'invasion de Chypre par les Mamelouks, en août 1426, Antoni de Fluvià, en accord avec les dignitaires du couvent, accorda au frère Joan Descarrigues, commandeur catalan de l'Espluga de Francoli et de Barbera institué

${ }^{41}$ AOM 373, ff. 42v-46v.

${ }^{42}$ AOM 282, ff. 117r-118r.

${ }^{43}$ Voir Tableau I.

${ }^{44}$ AOM 283, f. 30r.

45 J. Sarnowsky, Macht und Herrschaft, pp. 554-555. Nos recherches se sont appuyées sur une quarantaine de registres de la chancellerie de l'Ordre (AOM 346 à AOM 387).

${ }^{46}$ M. Bonet, Estructura gubernativa, pp. 64-70.

47 J. Sarnowsky, Macht und Herschaft, pp. 577-579. 
Lieutenant du maître pour toute l'Espagne, la mission de se procurer jusqu'à cent mille florins d'Aragon dans le royaume d'Aragon et le Principat de Catalogne pour y acheter des armes et contribuer à la défense de Rhodes $^{48}$. Il devait pour cela vendre des censals en obligeant, à titre d'hypothèque, les responsiones des commanderies destinées à garantir le paiement des pensions de ces censals.

Les censals constituaient un mode courant d'obtenir des fonds utilisé en Catalogne depuis le treizième siècle. Celui qui apportait un capital, dit l'acheteur (pour nous le prêteur), acquérait un droit, garanti par une hypothèque, à recevoir annuellement une pension en argent, droit acquis par achat et assorti d'un pacte de restitution ${ }^{49}$. Celui qui recevait les fonds, dit le vendeur (pour nous l'emprunteur), s'engageait à payer une pension annuelle d'un montant déterminé, obligation dont-il pouvait se libérer à tout moment en remboursant sans surcharge les montants qu'il avait reçus de l'acheteur. Ce droit de rachat, appelé lluïció, ne pouvait être exercé que par le vendeur. Le montant de la pension était proportionnel au capital apporté calculé selon un for (on évitait évidemment le terme intérêt) qui, pouvait osciller au XVe siècle entre $5 \%$ et $7 \%$. Le censal était présenté comme un acte d'achat et de vente donc parfaitement licite selon les canons de l'Ëglise.

On peut à priori s'étonner que les responsiones, qui avaient le caractère d'obligations pour les commandeurs de contribuer aux finances du Trésor de Rhodes, puissent être érigées en garantie. En fait, dans la constitution des censals les rentes de lieux bien déterminés de l'Ordre se voyaient assignées au paiement des responsiones que le Trésor renonçait donc à percevoir au profit des acheteurs de censals.

La mobilisation des 100000 florins d'Aragon par le commandeur Descarrigues en 1426 se passa très mal car le commandeur commit un abus de pouvoir ${ }^{50}$. Sachant que ces fonds étaient destinés à la défense de Rhodes, il se laissa convaincre par le roi Alphonse le Magnanime, dont il était un des proches, de verser au souverain l'intégralité de la somme, à charge pour le roi de mettre sur pied une flotte qui volerait au secours de Rhodes et de Chypre pendant quatre mois. En réalité, le roi avait bien plus en tête de trouver à financer des vaisseaux pour s'opposer aux Génois et conquérir le royaume de Naples et, par ailleurs, Fluvià avait momentanément écarté le risque Mamelouk par des négociations. Desgarrigues fut donc désavoué et le projet de flotte annulé. Le roi, dépité, prit possession de plusieurs commanderies catalanes et exigea le versement d'un subside de 61000 florins payé sur le capital des censals que l'Ordre dut alors vendre. Fluvià dut aussi aliéner deux lieux du prieuré de Catalogne dans le Roussillon et lever en France 10000 écus supplémentaires. La gestion rigoureuse du prieur de Catalogne et du receveur du Trésor permit ensuite de procéder à la lluïció de la plupart des censals et Rhodes put reprendre la perception des responsiones auxquelles le Trésor avait temporairement renoncé.

\subsubsection{Le recours aux censals par Jean de Lastic}

En 1444-1446, le maître Lastic, afin de faire face aux frais engagés pour résister aux Mamelouks qui avaient débarqué sur l'île et entrepris le siège de la ville, décida d'envoyer en Catalogne le Castellà d'Amposta, Joan de Vilagut, institué son lieutenant en Espagne, pour vendre des censals jusqu'à 50000 florins d'Aragon. Cette somme était destinée à rembourser d'une part 12100 ducats de Rhodes dus au marchand de Barcelone Miquel Ros; cette dette correspondait pour 10100 ducats à des

${ }^{48}$ ACA, AGP, Armoire 23, Parchemin núm. 1.

49 A. Garcia Sanz, El censal, pp. 286-307.

${ }^{50} \mathrm{P}$. Bonneaud, Le prieuré de Catalogne, pp. 278-281. 
remises de marchandises, des épices, et pour 2000 ducats à une avance en espèces. D'autre part, l'Ordre devait 8150 ducats à Ferrer Bertran, patron d'une grosse galère de Majorque, qui lui avait loué ou nolisé cette galère ${ }^{51}$. En effet, ces deux marchands faisaient partie de ces Catalans qui se livraient au commerce à Rhodes et dans le Levant. Ils s'y trouvaient au moment du siège et apportèrent leur concours, rémunéré, à la défense de l'Hôpital. Le taux de change appliqué pour le calcul du prix des censals était de 18 sous de Barcelone pour un ducat de Rhodes (18 sous et 6 deniers pour les 2000 ducats prêtés en espèces par Miquel Ros). Les créanciers faisaient certainement à ce taux un bénéfice de change. Par ailleurs, avec les autres fonds obtenus de la vente des censals, Vilagut devait acheter de l'artillerie et des munitions. Mais sur le chemin, la galère dite des Catalans qui transportait le Castellà disparut avec 374 personnes à bord, au large de Malte en novembre $1444^{52}$.

Quelques mois plus tard, en 1445, Lastic convoqua à son de cloche à l'église Saint-Jean une assemblée des frères présents à Rhodes par laquelle il fit approuver une large délégation de pouvoirs à son neveu et sénéchal, Guillaume de Lastic, commandeur de Lyon, pour le représenter en Espagne et y vendre jusqu'à 50000 florins d'Aragon, et même plus, à for de 14000 pour 1000 , c'est-à-dire avec un intérêt de 7,14\%. La mission de Guillaume de Lastic fit l'objet de l'approbation du pape et d'une lettre d'appui du roi d'Aragon ${ }^{53}$. Le chapitre général de Rhodes d'Avril 1446 donna par ailleurs au receveur du Trésor dans la Castellania, le commandeur d'Huesca Antoni Pere Saplana, le pouvoir de vendre 20000 florins de censals supplémentaires ${ }^{54}$.

Plusieurs documents figurant dans un registre, hélas partiellement très abîmé, du notaire Antoni Vinyes consacré à l'Hôpital et conservé aux archives de la ville de Barcelone permettent de faire le point des censals vendus ${ }^{55}$. Guillaume de Lastic avait vendu à Miquel Ros, pour un prix de 10904 livres de Barcelone une pension de censal annuelle de 607 livres, 15 sous, 6 deniers, soit un taux d'intérêt de 6, 5\% et à Ferrer Bertran, pour un prix de 7335 livres, une pension de 407 livres, 10 sous, 2 deniers, soit un taux de 5,5\%. Le receveur Antoni Pere Saplana avait également vendu en date du 18 août 1446 à Joan Stella, marchand de Barcelone à Rhodes, une pension 11000 sous de Barcelone (soit 550 livres), pour un prix de 11000 livres, soit un taux d'intérêt de 5\%. Dans ce cas, le for avait été fixé à 20000 pour 1000 . Comment expliquer cette différence de for, donc de rémunération de l'acheteur Stella, par rapport aux deux censals précédents?

Stella avait prêté au maître 13147 ducats de Rhodes à une date antérieure non précisée ${ }^{56}$. Le montant dû, évalué à 12803 livres de Barcelone, 45 sous et 7 deniers incluait aussi 770 livres 18 sous, 7 deniers, montant des intérêts dus par le maître à la date de la vente du censal et calculés selon le for de 14000 pour 1000 admis comme règle générale par le maître. Il semble que Stella, en acceptant pour le censal un capital de 11000 livres inférieur à sa créance et le paiement annuel d'une pension de 550 livres, à un taux d'intérêt de $5 \%$ consentait à l'Hôpital des conditions plus avantageuses que celles des censals de Ros et de Bertran ${ }^{57}$. Le receveur Saplana fit

${ }^{51}$ J. Bosio, Dell'Istoria, pp. 220-221.

${ }^{52}$ ACA RC 2653, f. 61.

${ }^{53}$ Ces instructions et ces lettres d'appui sont reproduites dans AHCB, Arxiu notarial XVII, 3.

${ }_{54}$ AOM 358, ff. 153r-154v.

55 AHCB, Arxiu notarial XVII, 3.

56 Ibidem.

${ }^{57}$ En faisant même abstraction des intérêts déjà dus de 770 livres 18 sous et 7 deniers, le montant du prêt consenti par Stella de 13.147 ducats de Rhodes, converti en monnaie de Barcelone au taux habituel de 18 sous pour un ducat, se serait élevé à 11.832 livres et 6 sous. 
par ailleurs état de la satisfaction de l'Ordre envers Stella qui avait conclu ce contrat avec beaucoup de bonne volonté et de libéralité (...) et s'était contenté et satisfait des dites quantités ${ }^{58}$.

Il n'y avait évidemment pas eu de remise de fonds de la part de ces trois acheteurs de censals puisqu'ils étaient déjà créditeurs de l'Ordre. L'examen détaillé du censal de Joan Stella fait apparaître que les rentes qui garantissaient les responsiones étaient très soigneusement désignées et que les trois acheteurs étaient en droit d'en nommer le collecteur. Les universités des lieux d'origine de ces rentes, dans le prieuré de Catalogne et la Castellania d'Amposta, prêtaient serment d'allégeance seigneuriale devant notaire aux trois acheteurs qui pouvaient saisir leurs garanties à tout moment. Par ailleurs une pénalité de 10000 florins était exigible pour tout manquement aux contrats. Le Trésor, pour sa part renonçait chaque année à 1565 livres de Barcelone de responsiones, ce qui équivalait à environ $80 \%$ des responsiones ordinaires du prieuré de Catalogne, tant que la llüició, c'est-à-dire le remboursement du capital, ne s'était pas produit. En fait, le Trésor renonçait aux responsiones des deux prieurés dans leur totalité, celles qui n'étaient pas assignées aux pensions de censals devant servir à leur lliuició.

\subsubsection{Les censals de Milly et Sacosta}

Ce qu'on prit l'habitude de désigner comme censals de Barcelone ne cessa d'ailleurs d'augmenter puisque de nouvelles autorisations de ventes furent données en 1454 pour 34000 florins qu'étaient chargés de lever le marchand catalan Pere Benet d'une part et le commandeur aragonais, Juan Ram, résidant à Rhodes, envoyé en Catalogne à cet effet $^{59}$. En 1461, à la mort du maître Jacques de Milly et après l'élection de son successeur, Pere Ramon Sacosta, qui se trouvait alors en Catalogne, le lieutenant du maître, Adhémar du Puy, et les baillis conventuels, constatant que le Trésor ne pouvait honorer le paiement des lettres de change qu'il avait émises pour acheter des armes, des munitions et des vivres, demandèrent à Sacosta de lever 150 000 florins d'Aragon en vendant des censals ou en tirant de nouvelles lettres de change ${ }^{60}$. Sacosta parvint à vendre 32000 livres de Barcelone de censals. Pour le reste, il chargea trois commandeurs des prieurés de France, de Saint Gilles et d'Auvergne de se les procurer dans leurs prieurés par des ventes de rentes assignées aux responsiones, assorties de cartas de gracia, par des rentes viagères ou par des lettres de change ${ }^{61}$. En 1466 en dépit des lluïcions effectuées, c'est-à-dire des rachats, les censals de Barcelone s'élevaient encore à 50000 livres de Barcelone évaluées à 40 000 écus de France ${ }^{62}$.

\subsection{Les lettres de change}

Une autre manière de se procurer des fonds était le recours aux lettres de change. Raymond De Roover, dans son ouvrage bien connu sur ce moyen de paiement, a bien montré comment la lettre de change était acceptable par l'Église car elle

58 AHCB, Arxiu notarial XVII, 3 (folios non numérotés).

${ }^{59}$ AOM 364, ff. 136v-138r et 40v-141v.

${ }^{60}$ AOM 373, ff. 42v-46v.

${ }^{61}$ Ibidem, « tam per vestram vendicionem censsualium mortuorum redditum responsionum nobis et nostro communis thesauro pertinencium instrumentis tamen gracie perpetue redimendi mediantibus redditum viageriorum et quocumque contractu cambii seu contractibus cambiorum ».

${ }^{62}$ AOM 283, f. 30r. 
alliait à une opération de crédit une opération de change dans une autre monnaie ${ }^{63}$. La lettre de change entraînait l'intervention de changeurs qui prenaient des risques et elle échappait de ce fait à l'accusation d'être un prêt usuraire. Compte tenu de l'importance des échanges entre Rhodes et l'Occident, la lettre de change était évidemment un mode de transfert de fonds particulièrement souple et adapté dans des opérations de commerce international. Mais en raison de la crise financière elle était surtout devenue pour le Trésor un mode courant d'emprunter à Rhodes des sommes remboursées à terme à l'étranger.

Une analyse que nous avons effectuée sur une période de plus de cinquante ans entre 1423 et 1477 fait apparaître que 282 ordres de paiement à terme par lettres de change furent donnés par les maîtres ${ }^{64}$. Les montants devaient être payés en écus de France pour 212 d'entre eux, soit 75\%, par le receveur général de l'ordre à Avignon, 23 à Londres par le prieur ou le receveur d'Angleterre en livres sterling, 12 à Barcelone en livres de Barcelone, 7 à Séville en doblas de Castille, 6 à Gênes, le reste étant réparti entre 7 autres places financières comme Bruges ou Venise. Dans tous ces cas, le Trésor utilisait donc ses propres receveurs ou prieurs comme payeurs en leur indiquant généralement d'utiliser les disponibilités que leur procuraient les responsiones collectées par leurs soins.

La justification des paiements était la remise faite au Trésor de Rhodes par le bénéficiaire lui-même (dans $72 \%$ des cas) ou par un représentant d'une somme destinée à la subsistance et à la maintenance du couvent, pro sustentacione et manutencione nostri conventi. Les montants des sommes remises en ducats ou en florins de Rhodes n'étaient jamais indiqués mais il s'agissait de prêts remboursables dans une autre monnaie dont la durée variait entre six mois et deux ans. La rémunération des prêteurs résidait dans le gain prévu sur le change. Le montant de chaque lettre de change était fréquemment de plusieurs milliers d'écus.

Si les lettres de change n'étaient pas honorées par l'Ordre, elles pouvaient faire l'objet après protêt d'un recambio, d'une « rechange », par l'émission d'une nouvelle lettre payable à Rhodes ou sur une autre place. Il s'agissait là d'une bonne affaire car le marchand encaissait deux fois le bénéfice de change. En 1451, le maître informait le Valencien Lluís de Santàngel qui avait avancé à l'Ordre 6000 ducats payables à Avignon que si le paiement de la lettre faisait défaut, il pourrait faire un recambio sur Naples, Florence, Montpellier, Valence ou Barcelone ad damnum et interesse comuni Thesauri ${ }^{65}$.

Les bénéficiaires de ces lettres de change étaient des marchands dont la plupart se trouvait à Rhodes puisque $72 \%$ d'entre eux y effectuaient euxmêmes leurs remises de fonds. 169 lettres de change $(60 \%)$ avaient pour bénéficiaires des Catalans (dont 117 Catalans à proprement parler, 19 Majorquins et 33 Perpignanais $)^{66} ; 89(32 \%)$ concernaient des Italiens (70 Génois, 9 Florentins, 10 Vénitiens) et pour le reste il s'agissait surtout de Français. Le nombre des bénéficiaires était toutefois inférieur à ces quantités car plusieurs individus consentaient plusieurs prêts.

${ }^{63}$ R. De Roover, L'évolution de la lettre de change.

${ }^{64}$ AOM 346 à 387.

${ }^{65}$ AOM 363 f. $208 \mathrm{v}$.

${ }^{66}$ Nous avons regroupé comme Catalans des Perpignanais et des Majorquins, généralement précisés comme tels, mais on constate fréquemment qu'un même personnage figure avec une origine différente selon les documents. 


\subsection{Les prêts à intérêt des marchands à Rhodes}

D'autres prêts au Trésor n'entraînaient pas de remboursements en Occident, ni donc d'opérations de change, car ils étaient remboursables à Rhodes même. Ils étaient reconnus comme des prêts à intérêt, ad dampna et interesse, exposés donc à la réprobation ecclésiastique. Sans doute trouve t'on trace en 1438 de quelques prêts consentis à l'Ordre sans intérêt ni lucre (sine aliquo lucro vel interesse) par le marchand catalan Ferrer Bertran ainsi que par Suffredo Calvi, un citoyen de Rhodes, et par le vénitien Carolo Morosini ${ }^{67}$. Mais de tels cas n'étaient guère habituels et il s'agissait de personnages qui pratiquaient avec l'Ordre de fructueux négoces, des ventes de poivre pour Bertran ou le fermage de la savonnerie de Rhodes pour Morosini.

Comme pour les lettres de change nous avons analysé les 162 prêts contractés entre 1423 et 1477. La durée habituelle de ces prêts était comprise selon les cas entre 6 mois et 2 ans et parfois même jusqu'à 3 ans. Les fonds, remis à Rhodes au Trésor, pro sustentacione et manutencione nostri conventi, généralement par le prêteur lui-même (sauf dans deux cas) étaient remboursables à Rhodes à l'échéance. La bulla obligacionis qui rendait compte de chacun de ces prêts ne comportait normalement aucune autre quantité que celle de la somme due à l'échéance, libellée en ducats ou florins de Rhodes, sans mention du montant des intérêts ni des fonds effectivement reçus. Nous savons toutefois que les génois Cattaneo de Cattaneo et Gregorio Imperiale recevaient en 1427 pour un prêt de 3000 ducats à un an 330 ducats soit 11\% d'intérêt, un autre génois, Bartolomeo Doria prenait $17 \%$ en 1444 et $22 \%$ en 1448 et $1450^{68}$. Tel devait être habituellement le coût des crédits des marchands à l'Ordre.

96 prêts à intérêt, soit $59 \%$ du total, étaient consentis par des Catalans, dont 63 Catalans à proprement parler, 23 Majorquins et 10 Perpignanais, 33 prêts soit $20 \%$, l'étaient par des Italiens dont 23 Génois, 8 Vénitiens et 2 Florentins. Parmi les 33 autres, 30 étaient des Rhodiens, la plupart sous les magistères de Fluvià et Lastic et deux étaient des commerçants maures.

\subsection{Prêts dissimulés et autres formes de crédits}

Fluvià puis Lastic eurent recours à des achats ou à des ventes de marchandises à terme qui constituaient des prêts à intérêt déguisés. Les opérations sur du poivre furent nombreuses et je n'en citerai comme exemples que deux ou trois cas qui parlent d'euxmêmes, parmi les 54 transactions sur cette épice qui ont été relevées ${ }^{69}$. En 1436, le maître Fluvià acheta au marchand catalan Ferrer Bertran une quantité de poivre pour la somme de 2195 ducats de Rhodes payable à six mois. Ce poivre fut immédiatement revendu à d'autres marchands pour 2000 ducats, la différence entre ces deux prix coûtant à l'Ordre l'équivalent d'un intérêt annuel proche de $20 \%$ pendant six mois ${ }^{70}$. Une opération de plus grande envergure fut menée par Lastic lorsqu'il acheta, en 1453 au marchand maure de Valence établi à Alexandrie, Sidi Galip Ripoll, 110 quintaux de poivre au prix de 100 ducats le quintal alors que le prix courant de cette épice était alors de 60 ducats le quin$\operatorname{tal}^{71}$. Le montant impressionnant de cet achat, soit 11000 ducats, ne serait payable qu'un an et demi plus tard, le maître cherchant sans aucun doute à revendre auparavant et à encaisser au plus vite et au meilleur prix possible la quantité reçue.

\footnotetext{
${ }^{67}$ Z. Tsirpanlis, Anecdota, pp. 331, 332, 335, 341.

${ }^{68}$ AOM 347 f. 231r, AOM 356, f. 199r, AOM 361, f. 273r-v.

${ }^{69}$ J. Sarnowsky, Macht und Herrschaft, pp. 580-581.

${ }^{70}$ AOM 352, f. 163r.

${ }^{71}$ Z. Tsirpanlis, Anecdota, pp. 692-694.
} 
Au lieu d'achat puis revente du produit, les fonds pouvaient être obtenus immédiatement par une promesse de vente avec paiement anticipé. Le même Lastic s'était engagé en 1450 à fournir sous un an au marchand catalan Francesc Ferrer 32 quintaux et 50 rouleaux de poivre pour un prix de 50 ducats le quintal dont le montant total de 1625 ducats serait immédiatement encaissé par le Trésor ${ }^{72}$. Si l'Ordre n'avait pu alors se procurer le poivre, l'acheteur eût été remboursé au prix de 61 ducats le quintal. On manque d'éléments pour apprécier dans ces opérations sur le poivre la part de spéculation sur un produit sans doute devenu rare et la part de la nécessité de se procurer de la Trésorerie sans recourir ouvertement à des prêts usuraires.

Une autre source de trésorerie consista, dans la décennie 1460, à vendre au marchand vénitien Giovanni Martini, avec paiement partiellement anticipé et à prix réduit, l'intégralité de l'importante récolte de sucre de la commanderie Chypriote dont l'Ordre se réservait cependant 12 quintaux chaque année pour ses propres besoins. Les contrats avec Martini conclus pour une période de 5 ans furent signés en 1449 et prorogés jusqu'en 1459, puis renouvelés en $1464^{73}$. Le commandeur de Chypre devait prendre à sa charge le paiement des intérêts des sommes avancées et, pour cette raison, la pension annuelle qu'il devait verser au Trésor avait été réduite de 7500 florins à 4000 florins $^{74}$.

Enfin, à deux reprises en 1463 Sacosta se fit avancer, pro manutencione et sustentacione conventu, 680 puis 2280 florins par les tuteurs de deux mineurs. En contrepartie, le Trésor s'engageait à utiliser ces fonds pour nourrir et vêtir les pupilles pendant dix ans, puis à restituer alors ce qui resterait dû des fonds qu'il avait reçus ${ }^{75}$.

Au total, pour se procurer des fonds par l'emprunt, l'Hôpital avait recours à une panoplie de formules financières qui avaient cours en Occident lorsque les pouvoirs princiers ou les communautés urbaines se trouvaient confrontées à un endettement de plus en plus courant et important. Le Trésor de l'Ordre, grâce à son réseau de receveurs dans chaque prieuré, était particulièrement à même de contracter des prêts et de les rembourser s'il en avait les moyens. Les fonds pouvaient être trouvés auprès d'organisations bancaires et l'Hôpital fit appel en 1450 à la banque des Médicis, mais le commerce souvent florissant dans la deuxième moitié du XVe siècle alimentait en liquidités les demandes de prêts de toutes catégories. Les Hospitaliers de Rhodes ne firent pas exception à la règle en mettant à contribution les nombreux marchands établis ou en visite à Rhodes pour pratiquer le grand commerce dans le Levant.

\subsection{Le rôle financier des marchands, contesté mais indispensable}

\subsubsection{L'influence des marchands occidentaux à Rhodes}

Le port de Rhodes apparaissait comme une escale sure, bien protégée et bien administrée par les Hospitaliers, et constituait dans bien des cas la destination finale du voyage, en particulier pour les Catalans pour $80 \%$ de leur commerce dans le Levant dans la deuxième moitié du XVe siècle selon Mario Del Treppo ${ }^{76}$. Rhodes était alors devenue le centre du commerce catalan, une plaque tournante et un actif entrepôt où s'échangeaient les produits d'Orient et ceux du Levant. Claude Carrère a fait valoir à quel point Rhodes avait joué pour les marchands catalans le même rôle que Chio

${ }^{72}$ Ibidem, pp. 532-534.

${ }^{73}$ J. Sarnowsky, Macht und Herrschaft, pp. 495-496.

${ }^{74}$ AOM 380, ff. 195v-196r. L'importance des sommes réservées au paiement des intérêts donne à penser que Martini, outre les avances de paiement pour le sucre, consentait à l'Ordre d'autres prêts.

${ }^{75}$ AOM 374, ff. 95r-96r.

${ }^{76}$ M. del Treppo, Els mercaders catalans, p. 60. 
pour les Génois ${ }^{77}$. Il est indéniable que la présence de deux maîtres catalans à Rhodes au cours du XVe siècle, Antoni de Fluvià entre 1421 et 1437 puis Pere Ramón Sacosta entre 1461 et 1467 fut très favorable à un afflux accru des marchands catalans.

Nous avons répertorié 202 marchands occidentaux documentés comme ayant demeuré à Rhodes et ayant eu des relations commerciales ou financières avec l'Ordre au cours de la période d'une cinquantaine d'années étudiée, certains prêteurs consentant plusieurs prêts. Le nombre de marchands créanciers de l'Ordre était donc inférieur au nombre de créances qui s'élevait à 450. Sur l'ensemble de la période, $36 \%$ des marchands de l'échantillon furent des Italiens, dont 50 Génois, 15 Vénitiens et 8 Florentins, alors que $55 \%$ furent des Catalans.

On constate, que 55 des marchands qui composent le corpus accordèrent au moins deux prêts remboursables à Rhodes en ducats ou en Occident par lettres de change et 35 d'entre eux au moins trois. Ils apparaissaient comme particulièrement intéressés à participer au financement de l'Ordre dont ils devaient se sentir proches. Plusieurs résidaient à Rhodes, comme le Catalan Bartomeu de Parets ou le Génois Tobias Lomellino, et d'autres, du moins, s'y rendaient périodiquement car ils remettaient eux mêmes au Trésor les sommes qu'ils avançaient.

Ce «noyau dur» des marchands prêteurs, sous Sacosta, comptait 23 Catalans, y compris ceux de Perpignan et de Majorque, et 7 Génois $^{78}$. Le cas du patricien vénitien Andrea Cornaro, lié à l'Ordre par des intérêts communs à Chypre dans l'activité sucrière et par son rôle d'intermédiaire politique officieux avec la République des Doges, est un cas particulier de même que le sont les prêts conjoints des Florentins Giovanni Peruzzi et Gentile Bardi agissant certainement comme facteurs de leurs illustres maisons mères.

L'Ordre ne pouvait qu'être favorable à l'activité de ces marchands qui alimentaient de leurs prêts ses finances, lui fournissait éventuellement des galères armées en cas de danger et dont l'activité commerciale assurait son approvisionnement en draps, en céréales et autres denrées. Tous les produits faisant l'objet d'opérations commerciales, à l'entrée ou à la sortie de Rhodes, y compris le trafic d'esclaves, étaient soumis au paiement de la taxe du commercium, à la gabelle du vin et des biscuits et à d'autres taxes, détail que le maître n'omettait jamais de rappeler lorsqu'il émettait des droits d'entrée et de séjour à Rhodes.

La communauté des marchands était certainement influente et devait savoir se faire entendre; les relations avec l'Hôpital, qui leur offrait la sécurité et les attraits du port et de la ville de Rhodes, ne semblent guère avoir été conflictuelles. Cependant en 1457 le pape Calixte III se fit l'écho de leur mécontentement face au désir de l'Ordre d'établir une nouvelle gabelle ${ }^{79}$. En une autre circonstance, alors que l'Hôpital était confronté à la difficulté de défendre l'île de Cos fréquemment attaquée par les Turcs, les marchands firent savoir que si des renforts y étaient envoyés, comme le demandaient le commandeur et la population de l'île, ils considéreraient que la défense de Rhodes s'en trouverait affaiblie. Ils n'hésiteraient pas dans un tel cas à déserter l'île et il fut alors décidé de rapatrier sur Rhodes une partie de la population de Cos au lieu d'y envoyer des renforts ${ }^{80}$.

${ }^{77}$ C. Carrère, Barcelone, centre économique, p. 640.

${ }_{78}$ Relevons, parmi les Catalans Bartomeu de Parets, Bartomeu Bach, Marti Caralt, Jaume Sacalm, Francesc Riera, Joan Giger, Nicolau Vallobar, les Majorquins Pere de Pacs, Joan de Tagamanent, Francesc Vidal et les Perpignanais Pere et Francesc Pericolles, Miquel Giralt; parmi les Génois, Tobias Lomellino, Bartolomeo, Paulo et Giovanni Doria, Luigi de Grimaldi, Nicolau Piccamiglio.

79 ASV, Reg. Vat. 444, f. 23r.

${ }^{80}$ J. Bosio, Dell'Istoria, pp. 274-275. 


\subsubsection{L'arrêt des prêts usuraires par les marchands}

L'activité financière des marchands faisait cependant l'objet de critiques de la Papauté au nom de la condamnation de l'usure. En 1450, le pape Nicolas V déclara avoir appris que de nombreux frères de l'Ordre se livraient à Rhodes à des opérations de caractère usuraire ${ }^{81}$. Les statuts interdisaient pourtant aux frères de pratiquer des actes de commerce ${ }^{82}$. Il apparaissait que non seulement le maître et le Trésor recourraient à des prêts à intérêt mais qu'à titre individuel certains Hospitaliers de Rhodes empruntaient aux marchands, voire même peut-être finançaient avec eux des opérations commerciales. Le pape ordonna à trois dignitaires du couvent, le drapier, le turcopolier et le bailli du commercium de faire interdire aux marchands de telles opérations.

Mais surtout le recours excessif du Trésor aux avances de fonds fournies par les marchands représentait une énorme saignée qui absorbait une grande partie des responsiones. Il y eut pendant les sept ans du magistère du Catalan Pere Ramon Sacosta, entre 1461 et 1467, un véritable emballement des prêts consentis à Rhodes sous forme de lettres de change ou d'avances en ducats avec 210 prêts sur le total de 450 que nous avons relevés sur l'ensemble de la période étudiée. 160 prêts furent consentis au cours de ce laps de temps par des Catalans. Il semble que la présence sans doute accrue de ces marchands catalans fut mise à profit par leur compatriote Sacosta qui ne parvenait pas à obtenir des prieurés occidentaux le paiement des annates qu'il leur exigeait.

Devant l'échec de Sacosta à diminuer la dette, le pape Paul II, convoqua à Rome en 1466 un chapitre général des Hospitaliers. Il imposa, entre autres mesures, un moratoire de six ans sur les remboursements, l'annulation de tous les « intérêts, usure et charges » des dettes du Trésor et interdit à tous les créanciers de faire jouer les garanties qu'ils avaient sur les biens de l'Ordre ${ }^{83}$. Ce moratoire fut prorogé de deux ans en 1471 par Paul II puis de trois ans l'année suivante par son successeur Sixte IV ${ }^{84}$.

Une telle mesure compliqua sans aucun doute les relations du maître et du Trésor envers les marchands créanciers de l'Ordre. Il y eut certains refus purs et simples d'honorer les engagements pris ${ }^{85}$. Dans d'autres cas, comme par exemple pour Francesc Riera, Catalan, il y eut émission d'une lettre de change, payable en Avignon selon le temps et le terme contenus, spécifiés et déclarés dans les lettres et ordonnances faites par le pape Paul II et le chapitre général de Rome sur le fait des créanciers de notre Religion. Cette lettre de change en écus venait remplacer deux avances de fonds faites à Rhodes en ducats. Les lettres de change, nous l'avons vu, étaient mieux acceptées par l'Église mais, dans le cas cité, il semble que son paiement ne pourrait être honoré avant la fin du moratoire ${ }^{86}$. Il y eut ainsi quelques 80 lettres de change émises pour les deux seules années 1467 et 1468, payables presque exclusivement par le receveur général d'Avignon ${ }^{87}$. Dans la plupart des cas il s'agissait de transformation en lettres de change de prêts à intérêt antérieurs payables à Rhodes. Fréquemment les bénéficiaires en étaient les marchands Bartomeu de Parets, Catalan, et Tobias Lomellino, Génois, qui apparaissaient comme ayant pris auparavant à leur

\footnotetext{
${ }^{81}$ ASV, Reg.Vat. 393, ff. 102v-103v.

${ }^{82}$ ADHG, H 13, ff. $94 \mathrm{r}-\mathrm{v}$ et $107 \mathrm{v}$.

${ }^{83}$ AOM 283, f. 32v.

${ }^{84}$ J. Sarnowsky, Macht und Herrschaft, p. 542.

${ }^{85}$ AOM 74, f. 68r.

${ }^{86}$ AOM 377, f. 180r.

${ }^{87}$ AOM 377, ff. $178 \mathrm{r}-197 \mathrm{v}$ et $202 \mathrm{r}-207 \mathrm{v}$.
} 
charge le remboursement de prêts consentis à l'Ordre par d'autres marchands. Leur intervention permettait à l'Ordre de donner satisfaction à une partie de ses créanciers sans paraître enfreindre les interdits du pape. Le sénéchal du maître et commandeur de Chypre, John Langstrother, de la même manière, fut fait bénéficiaire d'une lettre de change de 3088 écus pour avoir payé de sa poche deux lettres de change du Catalan Martí Caralt arrivées à échéance ${ }^{88}$.

Il y a toutefois lieu de penser que le maître Orsini prit certaines libertés vis-à-vis du moratoire. Selon Bosio, dès 1467, devant les réclamations des créanciers il aurait donné instruction au frère Odinet Lamelin, receveur général à Avignon, de payer ceux-ci selon ses possibilités ${ }^{89}$. Deux ans plus tard il ordonnait à ce même receveur de lui faire verser 11000 florins qu'il avait lui-même remboursés à divers marchands, évitant ainsi des frais et des litiges ${ }^{90}$. L'émission de lettres de change ne cessa point mais à un rythme très réduit: 5 en 1469, 9 en 1470, 21 entre 1471 et 1476 . Seul 7 cas de prêts remboursables à Rhodes en ducats nous sont apparus. Il est donc clair que le chapitre général de Rome de 1466 avait mis un terme au financement de l'Ordre par un grand nombre de marchands.

Il faut mentionner quelques expédients auxquels recourut le Trésor. Devant la pression des besoins et le manque de ressources, certains Hospitaliers consentirent à l'Ordre des prêts sur leurs fonds personnels, tel le commandeur de Cantavieja, Pedro Fernández de Heredia, qui avança en 14701060 florins d'Aragon remboursables en cinq ans ${ }^{91}$. En 1472 Edoardo de Carmedino, institué commandeur de Cos, Leros et Calamos, accepta de payer d'un seul coup huit années de pension, soit 10000 florins de Rhodes ${ }^{92}$. Plus modestement, répondant à un cri d'alarme du grand commandeur devant l'absence de fonds et de moyens pour assurer la sécurité de Rhodes, 67 frères du couvent apportèrent leurs dons au Trésor en $1471^{93}$. Devant l'urgence, en 1475, le Trésor vendit même pour 216 marcs d'argent provenant de la sacristie du couvent et le maître Orsini fit don d'une partie de son argenterie pour 600 marcs $^{94}$.

\subsubsection{Le recours à deux marchands financiers: Bartomeu de Parets et Tobias Lomellino}

Malgré des efforts certains pour améliorer la rentrée des responsiones et des annates et l'adoption d'autres mesures que nous évoquerons sous peu, il demeurait évident que l'Ordre ne pouvait se passer de l'aide financière des marchands de Rhodes. Changeant complètement d'approche, le Trésor, à partir du chapitre général de 1471, s'appuya presque exclusivement sur le Catalan Bartomeu de Parets et le Génois Tobias Lomellino. À la différence des nombreux marchands qui avaient consentis des prêts dans les années précédentes, ces deux personnages, qualifiés de marchands dans les textes, ne paraissent pas avoir fait commerce de marchandises. Sous une forme ou sous une autre, ils consentirent, chacun de leur côté, des prêts importants au Trésor entre 1463 et 1475, soit 28861 écus, 8400 ducats de Rhodes, 3000 doblas de Castille et 1325 livres sterling pour Parets, et, pour Lomellino, 24113 ducats, 1.000 ducats de Rhodes, 21300 doblas de Castille, 130 livres sterling, 832 florins de Sienne et 500

\footnotetext{
${ }^{88}$ AOM 378, f. 180r.

${ }^{89}$ J. Bosio, Dell'Istoria, p. 314.

${ }^{90}$ AOM 379, f. 190r.

${ }^{91}$ AOM 380, f. 167r.

92 Ibidem, f. 182 r.

93 AOM 374, ff. 59r-61v.

${ }^{94}$ J. Bosio, Dell'Istoria, pp. 349, 352.
} 
ducats de Venise. Cette diversité dans les lieux de paiement des lettres de change et dans les monnaies montrent d'une part qu'ils intervenaient au nom de prêteurs qui ne venaient pas à Rhodes eux-mêmes pour y formaliser leurs prêts et d'autre part qu'ils avaient une activité financière ailleurs qu'à Rhodes. Ainsi les montants importants payables à Séville en doblas, pour le Génois Lomellino, étaient destinés à son parent Lorenzo Lomellino, probablement son associé dans le grand port andalou où les marchands génois abondaient.

Les deux marchands avaient aussi en commun d'avoir obtenu le fermage de monopoles de l'Ordre, pour Parets la gabelle de $2 \%$ du commercium de 1459 à 1471 et celle dite du vin et des biscuits (vini et biscotti) de 1461 à 1471 et, pour Lomellino, celle du savon, la saponaria de 1463 à $1472^{95}$. L'un et l'autre étaient résidents de Rhodes et jouissaient de la confiance de l'Ordre et de ses dignitaires. Parets avait reçu la garde des joyaux du maître Sacosta et, après la mort de celui-ci à Rome où le chapitre général venait de se terminer, il vint remettre au représentants du Trésor qui s'y trouvaient encore 12 anneaux d'or ornés de diamants et 25 autres ornés de rubis, turquoise et diamants au titre des spolia du défunt dont il devait avoir la garde ${ }^{96}$.

Quelques mois après le chapitre général de 1471 où la décision fit l'objet d'une bulle, Parets et Lomellino, dorénavant associés, furent désignés par le maître, en son nom et en celui du Trésor, comme procureurs, receveurs, acteurs et facteurs du couvent et du Trésor avec pouvoir de réclamer, exiger, recevoir récupérer et tenir en dépôt de tous les receveurs et procureurs du Trésor institués partout dans le monde toutes sommes et quantités assignées et députées pour le soutien et la maintenance du couvent à l'exclusion toutefois des fonds destinés au règlement des dettes ${ }^{97}$. Pendant les cinq ans précédant le chapitre général, le maître Orsini avait administré financièrement le couvent mais ce mandat n'avait pas été renouvelé et l'administration des finances avait été restituée au grand commandeur et au collège de huit auditeurs, un par Langue, qui partageait ses pouvoirs. C'est cet organe collégial qui chargea Parets et Lomellino de répondre aux besoins de Trésorerie du couvent en utilisant les fonds que devaient leur procurer les différents receveurs de l'Ordre au titre des responsiones, annates et autres revenus. Mais il était prévisible que ces fonds ne leur parviendraient pas avec toute la fluidité voulue et que leur propre assise financière leur permettrait, si nécessaire, de faire des avances au Trésor. L'opportunité s'en présenta en 1473 lorsque les deux associés prêtèrent ensemble à l'Ordre l'équivalent de 7285 écus remboursables à un an à Avignon et de 4537 livres sterling payables à Londres sous huit mois ${ }^{98}$.

La situation des deux compères était d'autant mieux assurée qu'ils reçurent conjointement en fermage en 1472 le droit du commercium pour trois ans, renouvelé pour six ans supplémentaires en 1475, pour un prix annuel de 3333 florins et 7 aspres ${ }^{99}$. Ils se virent également attribuer pour trois ans la gabelle du vin et des biscuits au prix annuel de 7500 florins $^{100}$. La perception de ces taxes leur procurait de la trésorerie dont ils restituaient chaque trimestre une part au Trésor pour qui mieux valait compter sur deux financiers prospères et avisés que solliciter d'un grand nombre de marchands des prêts que condamnait l'Église.

Mais le rétablissement de l'équilibre par la diminution sinon par l'apurement de la dette supposait d'augmenter les rentrées de fonds.

\footnotetext{
95 AOM 374, ff. 179r et 181v; AOM 377, ff. 220v-221r.

${ }^{96}$ AOM 376, f. 205r.

97 AOM 381, ff. 148v-149v.

${ }^{98}$ AOM 381, ff. 193r-194v.

${ }^{99}$ AOM 381, ff. 195r-196v et f. 207r.

${ }^{100}$ AOM 74, f. 128v.
} 


\section{L'ACCROISSEMENT DES RESSOURCES}

\subsection{La surtaxation des commanderies}

J'ai déjà signalé qu'en temps normal le montant des responsiones annuelles, c'est-à-dire des versements annuels demandés par Rhodes aux commandeurs pour contribuer à la subsistance du couvent, tournait autour $25 \%$ des rentes de chaque commanderie. Lorsque des situations exceptionnelles se produisaient, l'Ordre imposait des taxations supplémentaires et de même nature généralement qualifiés d'annates.

Fluvià ne leva pas d'annates mais il se procura des ressources supplémentaires en portant à deux ans au lieu d'un seul la durée des vacantes pendant laquelle les revenus des commanderies qui se libéraient étaient réservés au Trésor ${ }^{101}$. La pratique des annates se généralisa après la première attaque des Mamelouks contre Rhodes en 1440, d'abord sous le forme d'une contribution exceptionnelle appelée le quinquenium équivalent au cinquième des rentes brutes des commanderies, y compris celles des chambres magistrales et prioriales, qui serait perçu pendant cinq ans par des receveurs spécialement désignés à cet effet. Afin de favoriser leur présence à Rhodes les commandeurs résidant au couvent ne seraient soumis annuellement qu'à un prélèvement supplémentaire de $10 \%{ }^{102}$. En 1444, après la deuxième attaque des Mamelouks, Lastic fit approuver le principe d'une annate égale à la totalité des rentes et à percevoir en deux ans, prélèvement confirmé par le chapitre général de Rome de 1446, bien que mal accueilli par les assujettis. Il fut ensuite décidé en 1450 puis en 1460 de faire payer en une seule fois une double responsio ${ }^{103}$.

Malgré le caractère répétitif et quasi permanent de ces contributions qui imposaient aux commandeurs de verser à Rhodes la moitié des rentes de leurs commanderies, l'endettement ne cessait de croître. Lors du chapitre général de 1462 le maître Sacosta fit approuver la mesure extrême de lever pendant trois ans une annate égale à la totalité des rentes de chaque commanderie, déduction faite de $25 \%$ de leurs montants, quantité jugée nécessaire pour assurer le bon fonctionnement d'une commanderie. Trop lourdement égalitaire, cette disposition provoqua une véritable levée de boucliers qui amena le pape Paul II à convoquer à Rome le chapitre général de 1466 et à prendre en mains un plan de redressement ${ }^{104}$. Il fut alors décidé de lever chaque année pendant cinq ans une demi-annate, c'est-à-dire 50\% des rentes. Le prélèvement fut ensuite répété pour des périodes de trois ans en 1471 et 1475. Dans l'ensemble, la crise militaire et financière avait donc eu pour premier résultat de doubler de façon durable les prélèvements imposés aux commandeurs.

Les commandeurs n'avaient jamais été de bons payeurs, au point qu'au terme de responsiones était toujours associé celui d'arriérés, rendant compte de l'insistance que devaient déployer les receveurs du Trésor dans les prieurés. Les différents maîtres s'employèrent, avec des fortunes diverses, à mettre plus de rigueur dans la perception des contributions pendant la crise. Chaque levée d'annates était suivie de l'envoi de dignitaires de Rhodes en Occident, chargés d'obtenir, avec l'appui du pape et des princes, les versements attendus de leurs commandeurs ${ }^{105}$. Ceux-ci s'exposaient, en cas de défaillance dans leurs paiements, à la perte de leur commanderie et même

\footnotetext{
${ }^{101}$ ADHG, H 13, f. 96r.

102 AOM 1649, Statut XII du chapitre de 1440.

103 P. Bonneaud, Els Hospitalers catalans, pp. 253-257, 259, 269.

104 Ibidem, pp. 339-340, 343-348.

105 Nicolas V, par exemple, en 1450 et 1452 , déclara condamner les frères qui ne payaient pas leur dû au Trésor de Rhodes (ASV, Reg. Vat. 393, ff. 99v-100v, 101v-102v, Reg. Vat. 397, f. 300r).
} 
de leur habit, c'est-à-dire leur condition de frères de l'Hôpital. Pour rendre plus aisé le regroupement des responsiones ou des annates et les faire parvenir plus rapidement à Rhodes, Lastic eut recours, à partir de 1450, aux services de la banque des Médicis et de ses facteurs ${ }^{106}$. La rentrée des contributions des commanderies en Occident et des rentes ou fermages perçus directement en Orient était devenu un enjeu majeur tant pour faire face aux dépenses du couvent que pour éteindre la dette.

\subsection{L'administration du couvent et du Trésor confiée au maître}

Une évolution dans les relations entre le maître et les organes du Trésor visa à mieux gérer et administrer le couvent, à mieux assurer les rentrées d'argent et également à accroître les ressources. À partir de 1429 avec Fluvià et jusqu'en 1480 et même au-delà, il devint fréquent de remettre aux mains du maître pour des périodes de trois à cinq ans le pouvoir d'administrer seul le couvent et d'en gérer les finances. Ces délégations se répétèrent en 1451 pour Lastic, puis pour Milly en 1454 et 1459 dans les deux cas pour trois ans. Sacosta en 1462, restitua la direction du Trésor au grand commandeur assisté des procureurs, du collège des huit auditeurs et du conservateur général, mais Paul II, au chapitre général de Rome de 1466, désavoua le maître auquel il imposa de prendre en mains la conduite des finances et la gestion du couvent tant que des demi-annates seraient levées. Le successeur de Sacosta, Orsini, gouverna sous ce régime jusqu'en 1471, puis en 1478 Aubusson assuma la même responsabilité.

En contrepartie, le maître gérait seul et comme il l'entendait toutes les possessions de l'Ordre dans le Levant et procédait seul aux nominations des officiers qui en étaient aux commandes. La prise en mains totale par le maître de la gestion du couvent répondait à un besoin d'efficacité en période de crise et, plus encore, visait à mettre entre parenthèse les éternels conflits entre les Langues sur la gestion des finances. Mais abandonner la gestion du couvent au maître avait aussi pour but d'amener celui-ci à contribuer financièrement à la prise en charge des dépenses et des dettes. Les revenus du maître en Orient et en Occident étaient importants et gérés sans reddition de compte au Trésor par le sénéchal. Ils n'étaient soumis à aucune responsio comme le fit souligner le pape lors du chapitre général de Rome en $1466^{107}$. La remise du pouvoir au maître s'accompagna donc du versement par celui-ci d'une partie de ses revenus pendant la durée de sa délégation, 12000 florins pour Fluvià et 12000 florins pendant deux ans pour Lastic ${ }^{108}$. En 1466, le chapitre général de Rome imposa à Sacosta d'apporter 8900 florins annuellement, ce qui était déclaré représenter la moitié des revenus du maître ${ }^{109}$.

Remettre la gestion des finances au maître sous-entendait sans doute que si les 54000 florins qui lui étaient assignés annuellement pour les dépenses de fonctionnement du couvent ne suffisaient pas, le maître ferait face aux dépassements sur ses propres ressources. En fait, Milly et Orsini se virent acculés à réclamer au Trésor des sommes qu'ils avaient dépensées et qu'ils estimaient leur être dues. Les conflits se multiplièrent et les délégations de ces maîtres se transformèrent en de continuels débats entre le Trésor qui avait repris tout son poids et un maître appauvri ${ }^{110}$. A sa mort, la dépouille d'Orsini consistait en de lourdes dettes à des tiers que le Trésor dut honorer ${ }^{111}$.

\footnotetext{
${ }^{106}$ ASV, Reg. Vat. 397, ff. 295r-298r.

${ }_{107}$ AOM 283, f. 33v.

108 AOM 363, f. 226v-228r.

109 J. Sarnowsky, The rights of the Treasury, pp. 269 et 272 et AOM 283, ff. 33r-33v.

110 Voir, pour Milly, AOM 366, ff. $163 \mathrm{v}-164 \mathrm{v}$ et pour Orsini, J. Bosio, Dell'Istoria, vol. II, pp. 328-329.

111 J. Bosio, Dell'Istoria, p. 384.
} 


\subsection{Autres apports}

\subsubsection{Augmentation et utilisation des taxes}

Une source traditionnelle de ressources était la taxe du commercium qui grevait toutes les marchandises tant à l'entrée qu'à la sortie de Rhodes au taux habituel de $2 \%{ }^{112}$. Après l'invasion de Chypre par les Mamelouks, Fluvià avait fait porter pour dix ans le taux de la taxe à $8 \%$, mesure prorogée de dix ans de plus par Lastic en $1439^{113}$. Ramené ensuite à $2 \%$ et remis en fermage en 1472 aux marchands Bartomeu de Parets et Tobia Lomellino pour un prix annuel de 3333 florins et 7 aspres il avait été décidé par les chapitre généraux de 1466 et 1471 que le produit du commercium serait consacré à la construction et à la réparation des murailles de Rhodes ${ }^{114}$.

Une autre taxe traditionnelle était la gabella vini et biscotti mise en fermage en 1447 à Zorzi Londachi et compagnie pour 6000 florins avec obligation pour le fermier d'armer la galère de la garde et d'y maintenir 164 marins $^{115}$. Cette même exigence d'utiliser la gabelle pour l'armement de la galère fut maintenue par le chapitre général de 1471, qui assigna aussi cette même fonction à une autre taxe récente, la gabella ponderis farinae ${ }^{116}$. Sacosta avait en effet fait adopter cette mesure, applicable à toute livraison de froment au moulin, lors du chapitre général de 1462 en contrepartie de l'abandon de la servitudo marine, obligation pour de nombreux Rhodiotes de prêter leurs services à bord des bâtiments de l'Ordre. Il imposa également une taxe de $2 \%$, dite Catene et Porto Rodi, sur la valeur de toutes les marchandises transitant par le port de Rhodes, dont le produit était destiné à financer les fortifications ${ }^{117}$.

Ainsi, la multiplication de ces taxes accompagnait-elle leur exclusive utilisation pour les frais entraînés par la défense du couvent.

\subsubsection{Les aides extérieures}

Le couvent bénéficia aussi de certaines aides des princes et de la papauté. Après la chute de Constantinople les papes Nicolas V, Calixte III, Pie II, Paul II et Sixte IV prirent des bulles de croisade et deux d'entre eux envoyèrent avec un succès éphémère des galères armées pontificales en Méditerranée orientale mais l'expédition préparée par Pie II ne partit point d'Ancône en raison de la mort du pape ${ }^{118}$. Non appuyées par la plupart des royaumes et principautés d'Occident, ces tentatives de croisades furent des échecs. Le passé et la mission de l'Hôpital le faisaient considérer comme le fer de lance de la papauté dans la lutte contre les infidèles alors qu'il n'en avait nullement les moyens tant militaires que financiers.

Sollicité par la papauté, en 1454, le chapitre général de Rhodes avait décidé de consacrer 20000 florins à la participation de l'Hôpital avec quatre galères armées à toute croisade que le pape ou des princes chrétiens lanceraient contre les Turcs. Lors

112 Sur les différentes taxes imposées à Rhodes, voir J. Sarnowsky, Macht und Herrschaft, pp. 447-454.

113 Z. Tsirpanlis, Anecdota, doc. 99, pp. 359-360.

114 AOM 283, f. 90r.

115 J. Sarnowsky, Macht und Herrschaft, doc. 29, pp. 646-647.

116 AOM 283, f. 89r.

117 J. Bosio, Dell'Istoria, p. 285.

${ }^{118}$ Les études sur la papauté et les croisades du XVe siècle sont nombreuses. Signalons celle de N. Housley, The Later Crusades. 
de la croisade menée par Calixte III (1455-1458), Rhodes servit de base à l'expédition et participa à l'opération, alors que le couvent était plongé en pleine crise financière ${ }^{119}$. Quelques vingt ans plus tard l'apport de l'Ordre à la ligue de Venise du pape et du roi de Naples contre les Ottomans se limita à deux galères ${ }^{120}$.

La papauté était consciente de la situation périlleuse qui était celle de Rhodes face aux Ottomans et de la détresse du Trésor de l'Ordre. Sans accorder à celui-ci un plan d'aide financière digne de ce nom, elle le fit bénéficier par l'intermédiaire des rois de France et d'Angleterre d'une partie des décimes ecclésiastiques et des indulgences qu'elle leva pour la croisade. Ainsi, sous le pontificat de Nicolas V, les indulgences vendues en France et en Angleterre à l'occasion du Jubilé pontifical de 1450 furent versées à Rhodes pour être consacrées à sa défense ${ }^{121}$. De même en 1457 , le roi de France Charles VII fit don de 16000 écus, prélevés sur les décimes levées au royaume de France pour la croisade ${ }^{122}$. Enfin, en 1477, son successeur Louis XI, obtint du pape Sixte IV l'autorisation de vendre des indulgences dans son royaume à l'occasion d'un nouveau Jubilé dont le produit serait exclusivement consacré à la défense de Rhodes et plus spécialement aux fortifications de la ville ${ }^{123}$. Les textes dont nous avons eu connaissance ne rendent compte, à l'exception près du subside de Charles VII, ni de l'importance de ces fonds, ni de l'utilisation qu'en fit l'Ordre.

Parmi les princes d'Occident, seul le duc de Bourgogne Philippe le Bon montra par des actes une disposition réelle à secourir Rhodes. En 1444, il envoya le chevalier Geoffroy de Thoisy avec plusieurs bâtiments participer à la défense du couvent contre les Mamelouks. En 1465, il fit une donation de 10000 écus que le maître Sacosta utilisa pour mener à bien la construction du fort Saint Nicolas, situé à l'entrée du port de Rhodes et dont le rôle fut essentiel dans l'échec des Ottomans lors du siège de la ville en $1480^{124}$.

Pour utiles que fussent ces donations et quelques autres, elles étaient bien insuffisantes pour permettre de faire face aux dépenses indispensables et au règlement de la dette. Il fallait encore améliorer la gestion financière du couvent et s'attaquer à de la réduction des frais et des dépenses.

\section{PLUS DE RIGUEUR DANS LA PRÉVISION ET DANS LE CONTRÔLE DES DÉPENSES}

\subsection{La transparence face à la crise}

Au fur et à mesure que l'Ordre s'enfonçait dans la crise on assista à un progressif mais incontestable effort de clarté, de transparence et de rigueur dans l'analyse des comptes et la recherche des remèdes. Dans un premier temps, lors du chapitre général de 1449 présidé par Lastic, un montant fut fixé pour le total des responsiones ordinaires que le Trésor devait percevoir en Occident en temps normal, soit 50000 florins de Rhodes, que l'on estima équivaloir à $25 \%$ des rentes totales de l'Ordre. Mais, comme il n'y avait jamais eu de recensement systématique des rentes des commanderies depuis celui fort partiel de 1373, il fut décidé d'organiser des visites sur place

\footnotetext{
119 P. Paschini, La flotta di Callisto III; M. Navarro Sorni, Alfonso de Borja.

${ }^{120}$ AOM 282, f. 15v et AOM 74, f. 13r. Aussi J. Bosio, Dell'Istoria, pp. 334-335.

${ }^{121}$ C. Marinescu, La politique orientale, p. 192; ACA, RC. 2557, ff. 10v-11v.

${ }^{122}$ AOM 367, ff. 214v-215r.

123 J. Bosio, Dell'Istoria, p. 373.

124 J. Paviot, Les ducs de Bourgogne, pp. 89-91, 101-102, 255.
} 
afin de pouvoir fixer le montant qui serait dû par chacune d'elles ${ }^{125}$. Il s'agissait là d'un travail de titans qui s'étala sur de nombreuses années puisque le premier état complet des rentes n'apparut que près de trente ans plus tard en $1476^{126}$.

Une seconde étape fut atteinte en 1451 lorsque Lastic assuma la gestion du couvent pendant trois ans ${ }^{127}$. Il fut alors décrété que le fonctionnement du couvent nécessitait en temps normal 54000 florins chaque année et que, pour y faire face Lastic recevrait, 18000 florins des rentes perçues par le Trésor à Rhodes, Chypre, Cos, Nissyros et Négrepont et 20000 florins remis par la compagnie Cosme de Medici, montant pris sur les responsiones d'Occident que cette compagnie était chargée de recevoir, le solde des responsiones étant consacrés au règlement des dettes. Le maître, de son côté, verserait 12000 florins chaque année, mais deux fois seulement et le Trésor s'engageait à lui restituer le cas échéant les sommes manquantes du fermage de Chypre si la perception était inférieure aux 24000 florins prévus. Par cet accord la notion d'un impératif budgétaire pour la gestion courante du couvent s'imposait ainsi que la prévision des origines des fonds nécessaires. Ce schéma fut également suivi lors de la remise de la gestion du couvent à Milly, le successeur de Lastic, en 1454 et 1459.

Après la mort de Milly, le chapitre général de 1462 présidé par Sacosta rendit au Trésor la responsabilité de l'administration financière du couvent auquel le maître ne souhaitait manifestement plus contribuer sur ses fonds personnels. L'assemblée se livra alors à une analyse détaillée de la dette estimée à 225342 écus de France en précisant la localisation des créances puis, après avoir décidé d'une annate de trois ans sur la totalité des rentes, l'assemblée adopta un plan de règlement des dettes et fixa l'origine des fonds nécessaires à la gestion du couvent pendant toute la période ${ }^{128}$.

Avec encore plus de rigueur et de détail le chapitre général de Rome de 1466, convoqué et conduit de mains ferme par Paul II, introduisit un schéma d'analyse et d'action financières qui allait servir de modèle aux chapitres généraux suivants de 1471 et 1475 et sur lequel il convient de s'attarder.

\subsection{L'exemple du chapitre général de Rome de 1466}

Paul II avait imposé au maître Sacosta, contre le gré de celui-ci, la convocation du chapitre général à Rome sous le contrôle de la Curie. Le produit de l'annate ne rentrait pas, les prêts usuraires se multipliaient et la dette s'accroissait alors que Sacosta voyait s'accumuler les critiques contre sa gestion au sein du couvent. En outre, le roi d'Aragon Jean II, en guerre contre la Generalitat catalane, réclamait au pape la destitution de Sacosta qui avait réussi à conserver sa dignité de Castellà d'Amposta et apportait son soutien aux insurgés en résistant aux partisans du roi dans ses deux commanderies de Miravet et Ascó ${ }^{129}$. Paul II ne destitua pas Sacosta qui mourut à Rome quelques semaines après la fin du chapitre, mais il avait pris en main le déroulement de la réunion en déléguant un archevêque et quatre évêques de la Curie pour la diriger, conjointement au maître et aux dignitaires de l'ordre. Les décisions prises firent l'objet de quatre bulles pontificales qui furent ensuite incorporées aux registres

125 AOM 501, f. 274r-v, statut 53, « de la nova responsió »; J. Sarnowsky, The Rights of the Treasury, p. 271.

126 J.M. Roger, Les différents types de commanderies, p. 36, note 66.

127 AOM 363, ff. 226v-228r.

128 AOM 382, ff. 11r-118v.

${ }^{129}$ P. Bonneaud, Els Hospitalers catalans, pp. 344 et 397-396. 
de la Chancellerie. Il s'agissait là d'une ingérence pontificale sans précédent dans la tenue d'un chapitre général, qui n'avait pas atteint une telle forme ni un tel degré lors du chapitre qu'avait convoqué à Rome Eugène IV en 1446.

La première bulle était consacrée à l'imposition des mesures en vue du règlement des dettes. Elle indiquait tout d'abord le montant en écus de France de ces dettes et les lieux où les créances devaient être honorées.

\section{Tableau 1}

\section{La dette de l'hôpital en 1466: montant et localisation}

En Écus du royaume de France: 287927 Écus

Situés à:

Avignon:

191120 Écus

66,4

Rhodes:

40457 Écus

14,0

Barcelone (censals)

Angleterre

40000 Écus

13,9

Castille

12250 Écus

4,4

4100 Écus 1,3

On prenait ensuite en considération le montant que les prieurés d'Occident devaient fournir pendant cinq ans, jusqu'au chapitre général suivant pour contribuer aux dépenses d'administration du couvent, soit 36000 florins de Rhodes par an. Convertie en écus, la somme nécessaire pendant cinq ans se montait à 90000 écus et, ajoutée au montant de la dette de 247929 écus, hors censals de Barcelone, elle faisait s'élever les besoins à 347924 écus ${ }^{130}$. En intégrant les 40000 écus des censals de Barcelone la dette s'élevait donc à 397924 écus. A partir de ce constat décision était prise de lever une demi-annate, c'est-à-dire, la moitié des rentes annuelles de chaque commanderie, dans chaque prieuré, pendant cinq ans ou, si son rendement était insuffisant, pendant six ans. La bulle prévoyait en détail ce devraient être les versements de chacun des prieurés pendant cinq ans, tant au titre des demi annates qu'à celui des arriérés des exercices antérieurs.

La deuxième bulle pontificale concernait l'administration du couvent, confiée à Sacosta ou à son successeur pendant la période de cinq ou six ans où serait imposée la demi-annate $e^{131} .54000$ florins étaient annuellement assignés au maître pour gérer le couvent pendant la période de sa délégation.

${ }^{130}$ Cette somme ne prenait pas en compte les censals de Barcelone qui seraient payées par les prieurés d'Espagne (40 000 écus) mais intégrait huit mille écus supplémentaires au titre des arriérés du prieuré de France. Nous ne nouds expliquons pas la différence de 2000 écus apparaissant dans la somme totale.

131 AOM 283, ff. 3r-36r. 


\section{Tableau 2}

\section{Origine des sommes annuelles remises au maître pour la gestion du Couvent de Rhodes (1466)}

\begin{tabular}{|c|c|}
\hline \multicolumn{2}{|c|}{ En florins de Rhodes } \\
\hline $\begin{array}{l}\text { Contribution personnelle du maître: } \\
\text { (soit la moitié des revenus du maître) }\end{array}$ & 8900 \\
\hline La moitié de la gabelle du vin de Rhodes: & 3000 \\
\hline Responsiones de Chypre, Cos et Négrepont: & 6100 \\
\hline $\begin{array}{l}\text { Contributions de } 13 \text { prieurés d'Occident: } \\
\text { (Langues de France, Provence, Auvergne et } \\
\text { Italie) }\end{array}$ & 36000 \\
\hline TOTAL & 54000 \\
\hline
\end{tabular}

\section{Source: AOM 283}

Les rentrées annuelles provenaient d'abord d'Occident pour 36000 florins pris sur les demi-annates des prieurés des Langues de France, de Provence, d'Auvergne et d'Italie. Le montant de ces versements était précisé dans chaque cas et devait être encaissé en juillet de chaque année par des procureurs spéciaux du maître dans des lieux d'Occident qui étaient Lyon, Avignon, Florence, Venise, Naples et Messine.

Les 18000 florins restants étaient perçus en Orient et consistaient d'une part en 8900 florins qu'apportait personnellement le maître, soit 50\% de ses rentes annuelles, tant en Occident qu'en Orient, d'autre part en 4000 florins, pension versée au maître par le commandeur de Chypre, puis 3000 florins soit la moitié de la gabelle du vin, 2.000 florins, montant des rentes de l'île de Cos, et 100 florins des responsiones de la commanderie de Négrepont. Par ailleurs, et en dehors des 54000 florins qui lui étaient assignés, le maître recevait l'autre moitié de la gabelle du vin, soit 3000 florins pour faire face à son obligation d'armer pendant quatre mois par an la galère de garde; il recevait aussi la taxe du commercium, de deux pour cent sur les exportations, tout ceci afin de faire face aux dépenses extraordinaires accoutumées, notamment les réparations et constructions des fortifications de la ville de Rhodes.

Si les sommes encaissées étaient inférieures aux prévisions le maître était autorisé à emprunter en émettant des lettres de change dont le remboursement et les frais incomberaient au Trésor. Enfin cette deuxième bulle se proposait d'encadrer et de réduire une grande part des dépenses.

La troisième bulle présentait les statuts adoptés par le chapitre ${ }^{132}$. Il s'agissait là de l'énoncé traditionnel des décisions de caractère institutionnel prises au cours d'un chapitre qui venaient s'intégrer à ce que l'on puisse appeler le corps législatif de l'Ordre. Par le passé, à côté des mesures concernant la vie religieuse, l'organisation et les règles internes de l'ordre, figuraient également les résolutions d'ordre financier mais le chapitre général de 1466 et ceux qui le suivirent avaient pris le parti de consacrer à celles-ci un traitement séparé.

${ }^{132}$ AOM 283, ff. 36v-42r. 
La quatrième bulle enfin établissait le moratoire de six ans dans le remboursement de la dette et la suppression des intérêts ${ }^{133}$.

\subsection{La réduction des dépenses}

Depuis 1450 les dépenses du couvent avaient donc été plafonnées à 54000 florins annuellement. On manque d'éléments de comparaison avec les périodes antérieures à l'exception d'un document de 1432 qu'il faut prendre avec prudence parce qu'il ne concerne qu'une seule année, pendant laquelle les dépenses s'étaient élevées à 65500 ducats soit près de 105000 florins $^{134}$. Sans aucun doute, l'effort demandé était très important. D'ailleurs, en 1475, le montant fixé pour les dépenses annuelles passa de 54000 florins à 66 400, sous la pression de l'augmentation du nombre des Hospitaliers au couvent, porté à 450.

Le nombre de frères fixé par les chapitres comme devant résider au couvent et dans les îles n'avait cessé d'augmenter: il était de 75 à l'arrivée de l'Ordre à Chypre en 1291, quantité qui fut rapidement portée à 150 pour demeurer inférieure à 300 au cours du XIVe siècle. Elle s'éleva à 335 en 1459 pour atteindre 350 , dont 300 chevaliers, en 1466 , puis 450 en 1471 et jusqu'à 550 au début du XVIe siècle.

Lastic, conscient du poids financier que représentait la présence de nombreux frères au couvent tant en salaires qu'en nourriture et en habillement, avait tenté de profiter de la trêve qu'il avait négociée avec les Mamelouks pour réduire le recrutement en Occident et les passages au couvent, en particulier en provenance du prieuré de Catalogne et de la Castellania d'Amposta ${ }^{135}$. Mais, après la chute de Constantinople, il était devenu impossible de diminuer les effectifs. Dès 1450, Lastic et son Conseil avaient en outre décidé qu'en raison de l'importance des dettes les commandeurs résidant à Rhodes ne pourraient plus être nourris par l'Ordre, ni recevoir de l'orge pour leurs chevaux. Ils ne devraient dorénavant compter que sur les revenus de leurs commanderies pour faire face à leurs besoins. Cependant, en raison de la pauvreté de certaines commanderies, il fut entendu que, pour celles rapportant moins de 100 florins annuellement, le Trésor compléterait le revenu de ces commandeurs jusqu'à cette quantité136.

Le chapitre général de 1466 fixa le détail des dépenses et imposa certaines baisses des frais liés à la présence des frères à Rhodes ${ }^{137}$.

\footnotetext{
133 AOM 283, f. 32v.

134 J. Sarnowsky, Macht und Herrschaft, p. 553; T.M. Vann, The Exchange of Information.

135 P. Bonneaud, Le prieuré de Catalogne, p. 171.

136 Z. Tsirpanlis, Anecdota, pp. 566-567 et 571-573.

${ }^{137}$ AOM 284, ff. 34v-35r,.
} 


\section{Tableau 3}

\section{La répartition des dépenses en 1466}

Montant annuel à la charge du maître, exprimé en florins de Rhodes (1 écu du royaume de France vaut 2 florins de Rhodes)

NATURE DES DÉPENSES

Église Saint-Jean

Infirmerie

Frais courants de défense*

Achats de nourriture

(Céréales, viande, légumes)

Orge pour les chevaux

Coût des auberges

(Tablées, serviteurs etc.)

Dépenses et salaires divers
MONTANT

1370

6000

10700

23000

2000

8000

5930

57000
$\%$

10,5

18,8

40,4

14,0

10,4

100,0

TOTAL

57000

*Incluent la garnison du château Saint-Pierre, le maintien d'une galère pendant 4 mois et les salaires des bombardiers

Source: AOM 283

De nombreux salaires diminueraient d'un tiers ou de moitié, à commencer par ceux des huit baillis conventuels et de leurs lieutenants appelés à passer de 300 à 200 florins annuellement pour les premiers et de 200 à 100 pour les seconds. Il faut dire que ces hauts dignitaires étaient généralement dotés de riches commanderies dans leurs prieurés d'origine. Des rémunérations seraient dorénavant perçues en pièces de drap et non plus en argent.

Malgré la prise en charge des frais d'hébergement par les commandeurs ayant des revenus supérieurs à 100 florins, les frais de table dans les auberges des Langues, avec ceux des camerarium et des autres frères s'élevaient à 8000 florins (12 800 florins en 1432). Le coût du ravitaillement en céréales, (y compris l'orge pour les chevaux à condition que ces derniers puissent supporter le poids d'un chevalier en armes) et en viande était estimé à 22700 florins au lieu de 24000 florins en 1432. Il faut sans doute prendre en compte le souci de l'Hôpital de constituer et conserver des stocks importants de grains et de nourriture dans la perspective de situation de siège de la ville. À l'issue du long siège de 1522, lorsque l'Hôpital dut partir de Rhodes, il restait encore six mois de vivres en réserve.

Si les dépenses occasionnées par le culte et par les réparations de l'église Saint-Jean, soit 1370 florins, ne donnèrent pas lieu à réduction, les frais relatifs à l'infirmerie devaient baisser de 6500 à 6000 florins et ceux concernant l'arsenal, de 1500 à 500 florins. Les dépenses affectées à la défense et figurant dans la bulle ne concernaient que la garnison du château Saint-Pierre pour 7200 florins et, pour 
3000 florins, l'armement par le maître de la galère de garde pendant quatre mois. Cert armement de la galère était en sus des 54000 florins accordés au maître, et financé par la moitié de la gabelle du vin, ce qui explique la somme totale de 57000 florins pour la répartition des dépenses en 1466. Il ne s'agissait là que des frais habituels à la charge du maître. Tant pour l'achat d'armes et de munitions que pour l'engagement d'embarcations armées et pour l'édification d'ouvrages militaires, il fallait compter sur d'autres fonds, des donations, les spolia des frères décédés, le produit du commercium et d'autres taxes ou de nouveaux emprunts.

\section{CONCLUSION}

L'importance et la durée de la crise financière des Hospitaliers de Rhodes, telle que nous venons de l'examiner, nous conduisent à un certain nombre de constats mais également à des interrogations auxquelles les sources et les travaux dont nous disposons ne nous permettent pas toujours de répondre. Tout d'abord, cette crise permet de mieux apprécier l'image et le poids militaire véritable de l'Ordre dans une Méditerranée orientale où s'imposait le pouvoir ottoman. L'Hôpital, après la disparition du Temple était apparu comme le seul bras armé de l'Église en Orient. Les rois et les princes lui reconnaissaient cette mission et qualifiaient les Hospitaliers d' « athlètes du Christ ${ }^{138}$. Cependant dès 1453, après la chute de Constantinople, Lastic, dans une missive étonnante de lucidité adressée à l'ensemble des prieurés d'Occident, avait pointé du doigt la grande solitude et le peu de moyens dont disposait l'Hôpital face à la volonté de conquête de Mehmed II. Les faits lui avaient donné raison avec l'échec des tentatives de croisade de trois papes et la perte par Venise de la plupart de son domaine en mer Égée.

Les maîtres et les dignitaires de leurs conseils se gardèrent sagement, du moins au cours de la période étudiée, de toutes provocations à l'égard des Turcs. Ils ne répondirent que mollement aux sollicitations de la papauté et envoyèrent même très discrètement des émissaires officieux à Constantinople pour tenter d'obtenir un accord de paix. Mais la nature de l'Ordre leur interdisait de se placer sous l'ombrelle des Ottomans. Ils adoptèrent donc, pendant ces années sombres une stratégie de survie, privilégiant à grands frais la fortification de l'île, le renforcement des effectifs militaires, le stockage de vivres et de munitions et le repli de la population dans les places fortes, minutieusement organisé. Cette politique explique le succès remporté face à la force déployée par l'ennemi qui attaqua Rhodes en 1480 avec 150 bâtiments, succès très médiatisé auprès des pouvoirs d'Occident par le maître Pierre d'Aubusson qui sut utiliser les moyens que lui offrait l'imprimerie pour redorer le blason de l'Ordre. Mais la vulnérabilité de l'Hôpital n'en était pas moins apparue extrême.

Un deuxième sujet concerne la manière dont les Hospitaliers gérèrent la crise financière. Celle-ci fut vécue intensément au couvent et débattue en permanence dans les conseils et les chapitres généraux mais l'oligarchie des hauts dignitaires qui entourait le maître fut fréquemment divisée. Les différents maîtres, à l'exception d'Antoni de Fluvià et de Pierre d'Aubusson firent l'objet d'attaques et de procès d'intention sur leur manière de gérer la crise, voire sur leur intégrité, sans que les documents nous éclairent véritablement sur la véracité des critiques dont ils étaient l'objet. Les papes Eugène IV puis Paul II prirent position contre Lastic en 1446 et Sacosta en 1466 respectivement et ils convoquèrent chacun un chapitre général à Rome. L'intervention pontificale de 1466 permit véritablement d'aborder tous les aspects de la crise et imposa un plan de redressement qui fit assez rapidement sentir ses effets: en 1478, la

${ }^{138}$ S. Pauli, Codice diplomatico, vol. II, p. 135. 
dette avait diminué de plus de moitié et de $80 \%$ en 1489. Cette évolution était d'autant plus remarquable que le siège de 1480 et plus encore les tremblements de terre de 1481 avaient causé des destructions telles qu'elles entraînèrent immédiatement des travaux très importants et un remodelage complet des fortifications.

Une question vient évidemment à l'esprit, celle de l'insuffisante mobilisation des commanderies d'Occident qui, au nombre proche d'un millier, semblaient devoir constituer, avec la perception de leurs rentes, un grand réservoir de liquidités. En l'absence de données fiables sur les rentrées des responsiones et des annates, l'envoi constant de dignitaires du couvent chargés de la mission d'exiger des commanderies les versements auxquels elles étaient tenus ainsi que les interventions auprès du pape et des princes pour qu'ils fassent pression en ce sens sur les propres commandeurs de l'Ordre constituent des indices certains de la difficulté à percevoir ces sommes. Il y eut au cours de la période, dans certains prieurés des raisons objectives à ces difficultés, par exemple en France et en Provence la destruction de nombreux biens de l'Ordre à la suite de la Guerre de Cent Ans ou des exactions des bandes armées qui sillonnèrent la Provence. En Catalogne et de l'Aragon l'appropriation de commanderies par Jean II lors de la guerre civile catalane priva le Trésor de ressources appréciables ${ }^{139}$. Mais notre opinion est que d'une manière générale, l'absence, lorsqu'ils étaient à Rhodes, des commandeurs de leurs commanderies, remises par eux à des fermiers ou à des membres de leurs familles, rendait beaucoup plus difficile de percevoir régulièrement les contributions qu'ils devaient à l'Ordre ${ }^{140}$.

La crise financière à Rhodes fut évidemment causée par la situation politique et militaire consécutive à la belligérance de l'Ordre avec les Mamelouks et les Ottomans, mais certains aspects essentiels de son déroulement nous renvoient au contexte financier et commercial en Occident. Le recours par l'Hôpital aux nombreuses formes de crédit que nous avons décrites et en particulier l'envolée des emprunts auxquels recourut le Catalan Sacosta n'est pas sans rappeler l'endettement des pouvoirs centraux et des collectivités locales en Catalogne au cours du XVe siècle ${ }^{141}$. Surtout, l'Hôpital sut tirer profit de la position de Rhodes, au cœur du toujours prospère grand commerce d'Occident avec le Levant, pour retenir dans l'île les marchands italiens et catalans qui lui apportèrent leur aide financière, assurèrent l'approvisionnement des Hospitaliers et payèrent les taxes qui permirent de mieux fortifier la ville. Alors que l'état d'alerte militaire et le gouffre financier tourmentaient sans relâche le couvent, la politique commerciale de l'Ordre constitua son véritable succès au cours de cette époque difficile.

\section{BIBLIOGRAHIE CITÉE}

Batlle, Carme, L'expansió baixmedieval, segles XIII-XIV, dans Vilar, Pierre (dir.), Història de Catalunya, Barcelona, Edicions 62, 1987, vol. III, pp. 249-321.

Bonet Donato, Maria, Estructura gubernativa y fiscaldad en la Orden del Hospital en la Corona de Aragón bajomedieval, dans Izquierdo Benito, R.; Molero García, J.; Ruiz Gomez, F. (eds.), La Orden Militar de San Juan en la Península Ibérica durante la Edad Media, Actas del Congreso internacional celebrado en Alcázar de San Juan los días 23, 24 y 25 de octubre de 2000, Alcázar de San Juan, Patronato municipal de cultura, 2002, pp. 45-73.

\footnotetext{
139 Voir à titre d'exemple de l'absentéisme des commandeurs, P. Bonneaud, Els Hospitalers catalans, pp. 397-402.

140 Voir à titre d'exemple de l'absentéisme des commandeurs, P. Bonneaud, Dos encomiendas hospitalarias, pp. 129-163.

${ }^{141}$ C. Batlle, L'expansió baixmedieval, pp. 316-320.
} 
Bonet Donato, Maria, La Orden del Hospital en la Corona de Aragón. Poder y gobierno en la Castellania de Amposta (ss. XII-XV), Madrid, Consejo Superior de Investigaciones Científicas, 1994.

Bonneaud, Pierre, Le prieuré de Catalogne, le couvent de Rhodes et la Couronne d'Aragon, 1415-1447, Millau, Etudes et Communication Édition, 2004.

Bonneaud, Pierre, Dos encomiendas hospitalarias de la Segarra (Cervera y Granyena) a finales de la Edad Media, "Miscel-lania Cerverina" 19 (2009), pp. 129-163.

Bonneaud, Pierre, Els Hospitalers Catalans a la fi de l'Edat Mitjana. L'orde del Hospital a Catalunya i a la Mediterrània, 1396-1472, Lleida, Pagès editors, 2008.

Bosio, Jacomo, Dell'Istoria della sacra religione e illustrissima militia di San Giovanni Gierosolimitano, vol. II, Rome, Stamperia di Guglielmo Faciotti, 1629.

Carrère, Claude, Barcelone, centre économique à l'époque des difficultés, 1380-1442, Paris, Mouton et Cie., 1976.

Del Treppo, Mario, Els mercaders catalans i l'expansió de la Corona catalano-aragonesa, Barcelone, Curial Edicións, 1976.

Demurger, Alain, Chevaliers du Christ. Les ordres religieux militaires au Moyen-Âge XIe-XVIe siècle, Paris, Éditions du Seuil, 2002.

De Roover, Raymond, L'évolution de la lettre de change: XIVe-XVIIIe siècles, Paris, Armand Colin, 1953.

Gabriel, Albert, La cité de Rhodes (MCCCX-MDXXII), Paris, Éditions de Boccard, 1923.

Garcia Sanz, Arcadi, El censal, "Boletín de la Sociedad Castellonense de Cultura" 37 (1961), pp. 286-307.

Housley, Norman, The Later Crusades. From Lyons to Alcazar (1274-1580), Oxford, Oxford University Press, 1992.

Luttrell, Anthony, The Hospitallers at Rhodes: 1306-1421, dans Luttrell, Anthony, The Hospitallers in Cyprus, Rhodes and the West, 1291-1440, Londres, Variorum Reprints, 1978, I, pp. 278-313.

Luttrell, Anthony, Notes on Foulques de Villaret, Master of the Hospital, 1305-1319, dans Luttrell, Anthony, The Hospitallers of Rhodes and their Mediterranean World, Aldershot, Ashgate, 1992, IV, pp. 73-90.

Luttrell, Anthony, The Hospitallers of Rhodes confront the Turks, 1306-1421, dans Luttrell, Anthony, The Hospitallers of Rhodes and their Mediterranean World, Aldershot, Ashgate, 1992, II, pp. 80-116.

Luttrell, Anthony, The Building of the Castle of the Hospitallers at Bodrum dans Luttrell, Anthony, The Hospitallers State on Rhodes and its Western Provinces, 1306-1462, Aldershot, Ashgate, 1999, VI, pp. 143-161.

Luttrell, Anthony, The Hospitallers and the Papacy, 1305-1314, dans Luttrell, Anthony, Studies on the Hospitallers after 1306, Rhodes and the West, Aldershot, Ashgate, 2007, V, pp. 595-622.

Luttrell, Anthony, The Hospitallers and their Florentine Bankers: 1306-1346, dans Luttrell, Anthony, Studies on the Hospitallers after 1306, Rhodes and the West, Aldershot, Ashgate, 2007, VI, pp. 17-24.

Marinescu, Constantin, La politique orientale d'Alphonse V d'Aragon, roi de Napoles (1416-1458), Barcelone, Institut d'Estudis Catalans, 1994.

Navarro Sorni, Miguel, Alfonso de Borja, papa Calixto III, en la perspectiva de sus relaciones con Alfonso el Magnánimo, Valence, Institució Alfons el Magnànim - Diputació de València, 2005.

Nicolau d'Olwer, Lluís, Un témoignage catalan du siège de Rhodes en 1444, "Estudis Universitaris Catalans” 12 (1927), pp. 376-387.

ANUARIO de Estudios Medievales, 42/2, julio-diciembre 2012, pp. 501-534

ISSN 0066-5061, doi:10.3989/aem.2012.42.2.01 
Paschini, Pio, La flotta di Callisto III (1455-1458), “Archivio della società romana di storia patria" 103-105 (1930-1932), pp. 177-254.

Pauli, Sebastiano, Codice diplomatico del Sacromilitare Ordine Gerosolimitano, vol. II, Lucques, per Salvatore e Giandomenico Marescandoli, 1737.

Paviot, Jacques, Les ducs de Bourgogne, la Croisade et l'Orient (fin XIVe et XVe siècle), Paris, Presses de l'Université de Paris-Sorbonne, 2003.

Roger, Jean-Marc, Les différents types de commanderies du prieuré de Champagne au XVe siècle, dans Luttrell, A.; Pressouyre, L. (dirs.), La Commanderie, Institution des ordres militaires dans l'Occident médiéval, Paris, Éditions du CTHS, 2002, pp. 29-56.

Sarnowsky, Jürgen, Der Konvent auf Rhodos und die Zungen (lingue) im Johanniterorden (1421-1476), dans Nowak, Zenon H. (ed.), Ritterorden und Region: politische, soziale und wirtschaftliche Verbindungen im Mittelalter, Torun, Uniwersytet Mikolaja Kopernika, 1995, pp. 43-56.

Sarnowsky, Jürgen, Macht und Herrschaft im Johanniterorden des 15. Jahrhunderts, Verfassung und Verwaltung der Johanniter auf Rhodos (1421-1522), Münster, LIT Verlag, 2001.

Sarnowsky, Jürgen, The Oligarchy at Work. The Chapters General of the Hospitallers in the XVth Century (1421-1522), dans Balard, Michel (dir.), Autour de la première croisade: actes du colloque de la Society for the study of the crusades and the latin East, Clermont-Ferrand, 22-25 juin 1995, Paris, Publications de la Sorbonne, 1996, pp. 267-276.

Sarnowsky, Jürgen, The Rights of the Treasury: the Financial Administration of the Hospitallers on Fifteenth-Century Rhodes, 1421-1522, dans Nicholson, Helen (ed.), The Military Orders, Aldershot, Welfare and Warfare, 1998, vol. II, pp. 267-274.

Tsirpanlis, Zacharias, Anecdota Eggrapha gia te Rodo kai ti Noties Sporades apo to archeio Joaniton Ippoton, 1421-1453, Rhodes, Ekdosē Grapheiou Mesaiōnikēs Polēs Rodou, 1995.

Valentini, Roberto, L'Egeo dopo la caduta di Costantinopoli nelle relazioni dei Gran Maestri di Rodi, "Bulletino dell'istituto storico italiano per il medio evo e Archivio Muratoriano" 51 (1936), pp. 137-168.

Valentini, Roberto, Un capitolo generale degli Ospitalieri di S. Giovanni tenuto in Vaticano nel 1446, "Archivio storico di Malta" 7/2 (1936), pp. 133-168.

Vann, Theresa M., The Exchange of Information and Money between the Hospitallers of Rhodes and their European Priories in the Fourteenth and Fifteenth Centuries, dans Burgtorf, J.; Nicholson, H. (eds.), International Mobility in the Military Orders, Twelfth and Fifteenth Centuries, Cardiff, University of Wales Press, 2006, pp. 34-47.

Vatin, Nicolas, L'Ordre de Saint-Jean de Jérusalem, l'empire ottoman et la Méditerranée orientale entre les deux sièges de Rhodes, 1480-1522, Louvain - Paris, Éditions Peeters, 1994.

Fecha de recepción del artículo: septiembre 2010

Fecha de aceptación y versión final: marzo 2011 